\title{
Gravitational wave polarization from combined Earth-space detectors
}

\author{
Philippoz, Lionel ; Boîtier, Adrian ; Jetzer, Philippe
}

\begin{abstract}
In this paper, we investigate the sensitivity to additional gravitational wave polarization modes of future detectors. We first look at the upcoming Einstein Telescope and its combination with existing or planned Earth-based detectors in the case of a stochastic gravitational wave background. We then study its correlation with a possible future space-borne detector sensitive to high frequencies, like DECIGO. Finally, we adapt those results for a single gravitational wave source and establish the sensitivity of the modes as well as the localization on the sky.
\end{abstract}

DOI: https://doi.org/10.1103/physrevd.98.044025

Posted at the Zurich Open Repository and Archive, University of Zurich ZORA URL: https://doi.org/10.5167/uzh-172682

Journal Article

Published Version

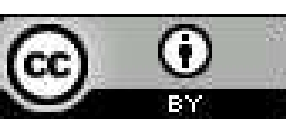

The following work is licensed under a Creative Commons: Attribution 4.0 International (CC BY 4.0) License.

Originally published at:

Philippoz, Lionel; Boîtier, Adrian; Jetzer, Philippe (2018). Gravitational wave polarization from combined Earth-space detectors. Physical review D, 98(4):044025.

DOI: https://doi.org/10.1103/physrevd.98.044025 


\title{
Gravitational wave polarization from combined Earth-space detectors
}

\author{
Lionel Philippoz, ${ }^{*}$ Adrian Boîtier, ${ }^{\dagger}$ and Philippe Jetzer ${ }^{*}$ \\ Physik-Institut, Universität Zürich, Winterthurerstrasse 190, 8057 Zürich
}

(Received 18 May 2018; published 16 August 2018)

\begin{abstract}
In this paper, we investigate the sensitivity to additional gravitational wave polarization modes of future detectors. We first look at the upcoming Einstein Telescope and its combination with existing or planned Earth-based detectors in the case of a stochastic gravitational wave background. We then study its correlation with a possible future space-borne detector sensitive to high frequencies, like DECIGO. Finally, we adapt those results for a single gravitational wave source and establish the sensitivity of the modes as well as the localization on the sky.
\end{abstract}

DOI: 10.1103/PhysRevD.98.044025

\section{INTRODUCTION}

Since the first detection of gravitational waves (GW) by the LIGO Collaboration, a total of five black hole mergers [1-5] has been observed so far [6], as have a neutron star merger visible through gravitational waves and all accessible frequency bands of the electromagnetic spectrum $[7,8]$. The results matched with Einstein's theory of general relativity (GR) up to measurement precision.

Until now, many tests of general relativity have been performed (e.g., the perihelion precession of Mercury, the geodetic precession and the Lense-Thirring effect by Gravity Probe B [9], or the weak equivalence principle by MICROSCOPE [10] to name only a few), and so far, they all agree with general relativity. Modifications to GR have been constrained by experiments, but there are still some possibilities that cannot be excluded; see Ref. [11] to get an overview. As we will discuss in the next part, one could, e.g., modify GR by adding a scalar or a vector field that only couples to the metric and therefore acts as correction to GR. These fields would allow additional polarizations to the two tensor polarizations, + (plus) and $\times$ (cross), predicted by GR. The scalar field leads to the emergence of the breathing $(b)$ and longitudinal $(l)$ polarizations, whereas the vector field is responsible for the appearance of the $x$ and $y$ polarizations.

The standard model of cosmology describes the creation of the Universe as an exponentially fast expansion of a quantum state. In quantum mechanics, no field or degree of freedom (d.o.f.) can be zero. If one now expands the Universe, the quantum fluctuations of the fields get macroscopic and create a homogeneous and isotropic background where all polarizations are excited equally.

\footnotetext{
pplionel@physik.uzh.ch boitier@physik.uzh.ch

\#jetzer@physik.uzh.ch
}

Using the electromagnetic spectrum, we can only observe events as far back as the cosmic microwave background. The neutrinos decouple a bit earlier and would allow us to see farther back in time, given we would figure out how to measure low-energy particles, which almost never interact. If we could, however, measure a gravitational wave background (GWB), then we could test cosmological models way farther back in time. One expects that the gravitational waves decouple at the Planck time due to the weak coupling of the metric to the other fields. This might allow us to get information about quantum gravity and thus an energy scale that is far out of reach of modern particle colliders. Since one expects all polarizations to be excited in the GWB, this would serve as a test for GR or allow us to put constrains on alternative theories of gravity by checking the presence or absence of additional polarization modes in a given signal.

The second-generation ground-based detectors advanced LIGO and advanced VIRGO can detect GWs from binary black holes (BBHs) and binary neutron stars (BNSs) [7]. A similar detector is being built in Japan (KAGRA) [12], and another advanced LIGO is planned in the near future in India (IndIGO) [13]. With the Einstein Telescope (ET) [14], a cluster of three detectors arranged in an equilateral triangle with an arm length of $10 \mathrm{~km}$, there are plans to build a third-generation detector in Europe that is supposed to be about ten times more sensitive to a GW signal than the current generation.

Space-borne detectors are also on their way. LISA pathfinder was a success [15], which is very promising for LISA [16], a cluster of three satellites planned to be launched as the next ESA L3 mission. LISA will be put on a heliocentric orbit, at about $20^{\circ}$ behind the Earth. DECIGO $[17,18]$ was originally planned to consist of four clusters distributed in Earth orbit around the Sun, each forming a $1000 \mathrm{~km}$ equilateral triangle with three satellites. A scaleddown version with arms of only $100 \mathrm{~km}$, B-DECIGO [19], 
initially presented as Pre-DECIGO [20], has recently been proposed as a first generation of deci-Hz detectors. It is planned to revolve around the Earth at an altitude of $2000 \mathrm{~km}$.

The ground-based detectors of the second generation are not capable of detecting the gravitational wave background on their own, and it is unlikely that an improvement of about 1 order of magnitude in sensitivity would be sufficient. But if we combine the signals of all the detectors that are built to measure BBHs and BNSs anyway, then one could enhance the sensitivity by 3 to 4 orders of magnitude and thus get more restrictive constraints on the GWB or even detect it.

With its high- and low-frequency interferometers, the ET is designed to measure in a frequency range from $1.5 \mathrm{~Hz}$ to $10 \mathrm{kHz}$. It therefore makes perfect sense to cross-correlate its signals with the ones of any second-generation groundbased detector, or even DECIGO. The correlation between the ET and DECIGO has the advantage that their noises are very different, since the ET is Earthbased and DECIGO is in space and therefore does not have any seismic noise, for instance. A correlation of the ET or DECIGO with LISA would, however, be difficult since the designed sensitivity of the latter lies within the range $10^{-4}$ to about $1 \mathrm{~Hz}$, out of the frequency band considered for the ET/DECIGO.

Testing GR by using the gravitational wave background can be of interest due to its constant and isotropic nature. One does not have to extract complex waveforms in a combination of all the six possible polarizations from the strain, which may differ according to the modified theory considered. Note that, because of the isotropy of the GWB, one cannot distinguish between + and $\times$ tensor polarizations, or $x$ and $y$ vector polarizations, but it is nevertheless possible to separate the three modes (tensor $T$, vector $V$, and scalar $S$ ), which can already give us information on the involved fields.

In the case of point sources, one can additionally determine the direction of the incoming GW on the sky as well as distinguish between the polarizations. However, this makes the calculation more complicated since we have to deal with 8 d.o.f. instead of 3 .

This paper is outlined as follows. In Sec. II, we recall some theoretical basics about polarization and summarize the derivation of the signal-to-noise ratio (SNR) for a polarization mode when combining multiple detectors in the case of a GWB, as done in Refs. [21,22]. We also derive the general expression of the power spectral density and the overlap reduction functions for detectors having arbitrary opening angles. In Sec. III, we apply those results in the case of the ET and consider its correlation with groundbased detectors. In Sec. IV, we introduce DECIGO in the detector network and investigate how the time-dependent sensitivity of a cross-correlation between DECIGO and Earth detectors can be used to distinguish the three polarization modes, as an alternative method to the maximum likelihood method on all detector pairs. Finally, we consider the case of point sources and derive the SNR for a single polarization and the variance on the incoming direction of the GW in Sec. V.

\section{THEORY AND METHODS}

In this section, we introduce the techniques used to calculate the sensitivities to GW polarizations of various combinations of detectors. We first give a short overview of GW and the notion of polarization in GR or alternative theories of gravitation, as well as the detection principle. We continue by extracting the signal of a correlation between two detectors. Then, we take multiple detector pairs and combine their signals in an optimal way to distinguish the polarizations and enhance the sensitivity.

The sensitivity is dependent on the noise power spectrum of the detector and geometry factors, which in the case of a gravitational wave background are the overlap reduction functions (ORFs). To calculate the sensitivity for a collection of detectors including the ET or DECIGO, we need to generalize the formula for the noise power spectral density to arbitrary opening angles, and we can simplify the expression for the ORFs for ground-based detectors, which comes in handy since many of the detectors we consider here are ground based.

\section{A. Polarizations of gravitational waves}

The linearization of the Einstein field equations leads to a linear wave equation for perturbations in the metric. Since the metric is required to be symmetric, the d.o.f. of a fourdimensional tensor of rank 2 are reduced from 16 to 10 . The Einstein equations are invariant under a change of reference frame, while the linearized version is only invariant under an infinitesimal change of coordinates, which reduces the d.o.f. to 6 . By choosing an orthonormal basis $(\hat{m}, \hat{n}, \hat{\Omega})$, where $\hat{\Omega} \| \vec{k}$ is the direction of travel [21], we can write a general solution as

$$
\begin{aligned}
h_{i j}(t, \vec{x}) & =\left(\begin{array}{lll}
h_{11} & h_{12} & h_{13} \\
h_{12} & h_{22} & h_{23} \\
h_{13} & h_{23} & h_{33}
\end{array}\right) e^{2 \pi i f\left(t-\frac{\hat{\Omega} \cdot \vec{x}}{c}\right)}+\text { c.c. } \\
& =\sum_{A} h_{A}(t, \vec{x}) e_{i j}^{A} e^{2 \pi i f\left(t-\frac{\hat{\Omega} \cdot \vec{x}}{c}\right)}+\text { c.c. }
\end{aligned}
$$

where the $e^{A}$ are the basis tensors of the possible polarizations we describe afterward and $h_{A}$ is the amplitude of the GW in the polarization $A$.

Therefore, we can have at most six polarizations. Since this is a vacuum equation in the case of unmodified GR, the equation is invariant under a gauge transformation on the fields $h_{\mu \nu} \mapsto h_{\mu \nu}^{\prime}=h_{\mu \nu}-\epsilon_{\mu, \nu}-\epsilon_{\nu, \mu}$, with $\square \epsilon^{\mu}=0$. This further reduces the d.o.f. to the two tensor polarizations + and $\times$. They are purely transversal waves, which enlarge 
distances in one direction and squeeze space in the orthogonal direction. The basis tensors of the tensor mode are given by

$e^{+}=\hat{m} \otimes \hat{m}-\hat{n} \otimes \hat{n}, \quad e^{\times}=\hat{m} \otimes \hat{n}+\hat{n} \otimes \hat{m}$.

We will now look at two representative examples of modifications of GR and their consequences on gravitational waves. Adding a scalar field to the Lagrangian is one possibility to modify GR [11]. A general scalar-tensor action can be written as

$S[g, \phi]=\frac{1}{16 \pi G} \int\left[R-2 g^{\mu \nu} \partial_{\mu} \phi \partial_{\nu} \phi-U(\phi)\right] \sqrt{-g} d^{4} x$.

This leads to the two scalar polarizations called the breathing mode $b$, since it stretches and squeezes space simultaneously in all transversal directions, and the longitudinal mode $l$, which is a purely longitudinal wave. Their basis tensors are given by

$$
e^{b}=\hat{m} \otimes \hat{m}+\hat{n} \otimes \hat{n}, \quad e^{l}=\sqrt{2} \hat{\Omega} \otimes \hat{\Omega} .
$$

Another possibility would be to add a vector field Lagrangian as

$$
\begin{aligned}
S[g, V]= & \frac{1}{16 \pi G} \int\left[\left(1+\omega V_{\mu} V^{\mu}\right) R-K_{\rho \sigma}^{\mu \nu} \nabla_{\mu} V^{\rho} \nabla_{\nu} V \sigma\right. \\
& \left.+\lambda\left(V_{\mu} V^{\mu}+1\right)\right] \sqrt{-g} d^{4} x
\end{aligned}
$$

with

$$
K_{\rho \sigma}^{\mu \nu}=c_{1} g^{\mu \nu} g_{\rho \sigma}+c_{2} \delta_{\rho}^{\mu} \delta_{\sigma}^{\nu}+c_{3} \delta_{\sigma}^{\mu} \delta_{\rho}^{\nu}-c_{4} V^{\mu} V^{\nu} g_{\rho \sigma},
$$

where the $c_{i}$ are coefficients that would have to be determined by experiments. This modification generates the two vector polarizations $x$ and $y$, which oscillate in the direction of travel and in one orthogonal to it. Their respective basis tensors are given by

$$
e^{x}=\hat{m} \otimes \hat{\Omega}+\hat{\Omega} \otimes \hat{m}, \quad e^{y}=\hat{n} \otimes \hat{\Omega}+\hat{\Omega} \otimes \hat{n} .
$$

We can finally express a general solution in terms of all six polarizations:

$h_{i j}(t, \vec{x})=\left(\begin{array}{ccc}h_{b}+h_{+} & h_{\times} & h_{x} \\ h_{\times} & h_{b}-h_{+} & h_{y} \\ h_{x} & h_{y} & h_{l}\end{array}\right) e^{2 \pi i f\left(t-\frac{\hat{\Omega} \cdot \vec{x}}{c}\right)}+$ c.c.

When a gravitational wave stretches or squeezes an arm of a Michelson interferometer, then one can observe a phase shift. This phase shift is larger if the amplitude of the wave is larger and if the detector arms are optimally aligned, given an incoming wave with a certain polarization. So, the signal in the detector can be written as

$$
h_{i j}(t, \vec{x})=D^{i j} \sum_{A} h_{A}(t, \vec{x}) e_{i j}^{A} e^{2 \pi i f\left(t-\frac{\hat{\Omega} \cdot \vec{x}}{c}\right)} .
$$

The detector tensor $D=\frac{1}{2}(\hat{u} \otimes \hat{u}-\hat{v} \otimes \hat{v})$ describes the orientations of the interferometer arms, given by the unit vectors $\hat{u}$ and $\hat{v}$, and the polarization of the wave can be written as a linear combination of the basis tensors $e^{A}$ described above. If we contract the two tensors, we get a scalar quantity, the angular pattern function, which describes the geometric dependence of the signal:

$$
F^{A}:=D^{i j} e_{i j}^{A}
$$

A GW thus produces one scalar signal in each detector, which means that we need to combine at least six detectors to distinguish them. In the case of a gravitational background, however, we expect a direction-independent signal. Therefore, one can only distinguish between the three modes (tensor, vector, and scalar); three independent signals are thus sufficient, but more signals would of course improve the sensitivity.

By using the matched filtering method, one can calculate the signal-to-noise ratio of a given signal. Since the ET project has declared a signal-to-noise ratio of at least 8 as their condition to accept an event as an actual signal [14], we set the SNR to 8 and calculate the minimal amplitude a gravitational wave needs to have to be recognized as a true signal by a certain collection of detectors and use this as a measure of their combined sensitivity.

The SNR is related to the false alarm rate $\alpha$ and detection rate $\gamma$ by [23]

$$
\mathrm{SNR} \geq \sqrt{\frac{2}{n}}\left(\operatorname{erfc}^{-1}(2 \alpha)-\operatorname{erfc}^{-1}(2 \gamma)\right)
$$

Once one has chosen a minimal signal-to-noise ratio, one has to choose either a false alarm or a detection rate. If we were to split our observation time $(T=1 \mathrm{yr}$ would be a realistic choice for a GWB observation) into small time intervals of, e.g., $4 \mathrm{~s}$ and do statistical tests on them, then one false alarm in 27,000 yr would be equivalent to a false alarm rate of $\alpha=\frac{4 s}{27000 \mathrm{yr}}=4.8 \times 10^{-12}$. This would give us about $n=7.8 \times 10^{6}$ time splits and result in a detection rate $\gamma \approx 1$ under the assumption of a SNR of 8 . The detection rate is related to the false dismissal rate $\beta$ by $\gamma=1-\beta$, which gives us a false dismissal rate of $\beta=3.3 \times 10^{-18}$.

We will now derive an expression for the SNR in terms of the GW signal and the detector noise. 


\section{B. Combined sensitivity of multiple detectors}

Since the signal of a GW is usually smaller than the noise, one can rely on two different techniques in order to get rid of the noise. First, we can multiply the Fourier transform of the signal with a suitable filter function, which turns out to be proportional to the signal, and integrate over all frequencies. This method is called matched filtering. Second, we can cross-correlate the strains $s_{I, J}=h_{I, J}+n_{I, J}$ of two detectors $I$ and $J$. Since the noises $n_{I, J}$ of the two detectors are not correlated between them and also not correlated to the signals $h_{I J J}$, we can get rid of the noise by taking the expectation of the Fourier transform (FT) of the complex conjugated strain $\tilde{s}_{I}^{*}$ of detector $I$ multiplied with the FT of the strain $\tilde{s}_{J}$ of detector $J$ :

$$
\mathbb{E}\left[\tilde{s}_{I}^{*} \tilde{s}_{J}\right]=\mathbb{E}\left[\tilde{h}_{I}^{*} \tilde{h}_{J}\right]+\underbrace{\mathbb{E}\left[\tilde{h}_{I}^{*} \tilde{n}_{J}\right]}_{=0}+\underbrace{\mathbb{E}\left[\tilde{n}_{I}^{*} \tilde{h}_{J}\right]}_{=0}+\underbrace{\mathbb{E}\left[\tilde{n}_{I}^{*} \tilde{n}_{J}\right]}_{=0} .
$$

Nishizawa et al. [21] used the matched filtering method on a cross-correlated signal and derived the SNR for a detector pair $(I, J)$, and we now shortly remind the reader of the result.

The energy density parameter $\Omega_{\mathrm{GW}}$ of the GWB can be written as a sum over all modes $M$, where each mode has two polarizations $M_{1}$ and $M_{2}$ as discussed previously:

$$
\begin{aligned}
\Omega_{\mathrm{GW}}(f) & =\sum_{M} \Omega_{\mathrm{GW}}^{M}=\sum_{M}\left(\Omega_{\mathrm{GW}}^{M_{1}}+\Omega_{\mathrm{GW}}^{M_{2}}\right), \\
M & =\left(\begin{array}{c}
M_{1} \\
M_{2}
\end{array}\right) \in\left\{T=\left(\begin{array}{c}
+ \\
\times
\end{array}\right),\right. \\
V & \left.=\left(\begin{array}{l}
x \\
y
\end{array}\right), S=\left(\begin{array}{l}
b \\
l
\end{array}\right)\right\} .
\end{aligned}
$$

The power spectral density $S_{h}^{M_{i}}$ of the polarization $M_{i}$ is related to its energy density parameter by

$$
\Omega_{\mathrm{GW}}^{M_{i}}(f)=\frac{2 \pi^{2}}{3 H_{0}^{2}} f^{3} S_{h}^{M_{i}}(f) .
$$

By assuming that only one mode $M$ is excited and using the ansatz $S_{h}^{M_{i}}(f)=h_{0, M_{i}}^{2} \delta\left(f^{\prime}-f\right)$, where $h_{0, M_{i}}$ is the amplitude of the polarization $M_{i}$, we get the sensitivity of the detector pair $(I, J)$ to the specific mode $M$,

$$
\begin{aligned}
\left(\mathrm{SNR}_{I J}^{M}\right)^{2} & =\frac{3 H_{0}^{2}}{10 \pi^{2}} \sqrt{T \int_{-\infty}^{\infty} \frac{\left(\Omega_{\mathrm{GW}}^{M}\left(\left|f^{\prime}\right|\right) \gamma_{I J}^{M}\left(\left|f^{\prime}\right|\right)\right)^{2}}{f^{6} P_{I}\left(\left|f^{\prime}\right|\right) P_{J}\left(\left|f^{\prime}\right|\right)}} d f^{\prime} \\
& =\frac{1}{5} \sqrt{T \int_{-\infty}^{\infty} \frac{\left(S_{h}^{M}\left(\left|f^{\prime}\right|\right) \gamma_{I J}^{M}\left(\left|f^{\prime}\right|\right)\right)^{2}}{P_{I}\left(\left|f^{\prime}\right|\right) P_{J}\left(\left|f^{\prime}\right|\right)} d f^{\prime}} \\
& =\frac{T}{5} \frac{\left(h_{0, M_{1}}^{2}+h_{0, M_{2}}^{2}\right) \gamma_{I J}^{M}(f)}{\sqrt{P_{I}(f) P_{J}(f)}},
\end{aligned}
$$

where $H_{0}$ is the Hubble constant, $T$ is the observation time, $P_{I, J}$ are the noise power spectral densities of the detectors $I$ and $J$, and $\gamma_{I J}^{M}$ is the overlap reduction function defined by

$$
\begin{aligned}
\gamma_{I J}^{M}(f):= & \frac{5}{2} \int_{\mathbb{S}^{2}}\left(F_{I}^{M_{1}} F_{J}^{M_{1}}+F_{I}^{M_{2}} F_{J}^{M_{2}}\right) e^{\frac{2 \pi i}{c} \hat{\Omega}_{0} \cdot \Delta \vec{x}_{I J}} \frac{d \hat{\Omega}}{4 \pi} \\
= & \rho_{1}^{M}(\alpha) D_{I}^{i j} D_{i j}^{J}+\rho_{2}^{M}(\alpha) D_{I, k}^{i} D_{J}^{k j} \hat{d}_{i} \hat{d}_{j} \\
& +\rho_{3}^{M}(\alpha) D_{I}^{i j} D_{J}^{k l} \hat{d}_{i} \hat{d}_{j} \hat{d}_{k} \hat{d}_{l},
\end{aligned}
$$

with $\alpha(f):=\frac{2 \pi f\left|\Delta \vec{x}_{I J}\right|}{c}$ and where the $\rho_{i}^{M}$ are linear combinations of the zeroth, second, and fourth spherical Bessel functions.

By requiring again $\mathrm{SNR} \geq 8$ for a $\mathrm{GW}$ with mode $M$ to be considered a true signal, we can rewrite (15) to get the minimal amplitude a GW would need to be detected as such:

$$
\left|h_{0}^{M}(f)\right|_{\min }=8 \sqrt{\frac{5}{T}}\left(\frac{\sqrt{P_{I}(f) P_{J}(f)}}{\left|\gamma_{I J}^{M}(f)\right|}\right)^{1 / 2} .
$$

If we have more than two detectors, we can use the maximum likelihood method to distinguish the polarizations. We then get a SNR with which we recognize a mode $M$, as derived by Nishizawa et al. [22]:

$$
\begin{aligned}
\left(\mathrm{SNR}^{M}\right)^{2} & =\frac{3 H_{0}^{2}}{10 \pi^{2}} \sqrt{T \int_{-\infty}^{\infty} \frac{\left(\Omega_{\mathrm{GW}}^{M}(f)\right)^{2} \operatorname{det} \mathbf{F}(f)}{f^{6} \mathcal{F}_{M}(f)} d f} \\
& =\frac{1}{5} \sqrt{T \int_{-\infty}^{\infty} \frac{S_{h}^{M}(f)^{2} \operatorname{det} \mathbf{F}(f)}{\mathcal{F}_{M}(f)} d f}
\end{aligned}
$$

Using the same ansatz as above, we get the minimal amplitude we require to not only detect a $\mathrm{GW}$ with mode $M$ but also distinguish its polarization, with a SNR of at least 8 ,

$$
\left|h_{0}^{M}(f)\right|_{\min }=8 \sqrt{\frac{5}{T}}\left(\frac{\mathcal{F}_{M}}{\operatorname{det} \mathbf{F}}\right)^{1 / 4}
$$

where the Fisher matrix $\mathbf{F}$ is obtained by summing over the Fisher matrices of all detector pairs $(I, J)$,

$$
F_{M M^{\prime}}(f)=\sum_{(I, J)} \int_{0}^{T_{\mathrm{obs}}} \frac{\gamma_{I J}^{M}(t, f) \gamma_{I J}^{M^{\prime}}(t, f)}{P_{I}(f) P_{J}(f)} d t,
$$

and $\mathcal{F}_{M}$ is the determinant of the minor one gets by removing the $M$ th row and column from $\mathbf{F}$.

\section{Optical read-out noise}

The quantum fluctuations of the laser cause a fundamental noise source in each detector that is statistically 
independent from the other detectors. The fluctuation in the number density of photons arriving at the detector causes a random fluctuation in the measured power and a fluctuation in the light pressure on the mirror, which causes the mirror to vibrate randomly. By increasing the laser power, the fluctuation in the number density increases in total but is less compared to the average, which causes the relative fluctuations in the measured laser power to decrease, but the pressure and therefore the fluctuations in the position of the mirror increase. One therefore needs to balance one effect against the other, which causes an uncertainty relation similar to the one arising from quantum mechanics. We are now going to derive the optical read-out noise based on Ref. [24], but for an arbitrary opening angle between the detector arms.

A Michelson interferometer with a Fabry-Perot cavity catches an additional term dependant on the frequency $f$ of the measured gravitational wave and on a pole frequency $f_{p}$, which is a characteristic of the cavity. The power recycling $C$ appears as a higher effective power, and the detector efficiency $\eta$ as a lower one, and we modifiy the input power $P_{0}$ as $P_{0} \mapsto \eta C P_{0}$.

The phase shift of a Fabry-Perot interferometer $\Delta \phi_{\mathrm{FP}}$ is related to the one of a Michelson interferometer without cavity $\Delta \phi_{\text {Mich }}$ by

$$
\begin{gathered}
\left|\Delta \phi_{\mathrm{FP}}\right|=\frac{2 \mathcal{F}}{\pi} \frac{\left|\Delta \phi_{\mathrm{Mich}}\right|}{\sqrt{1+\left(\frac{f}{f_{p}}\right)^{2}}}, \\
\left|\Delta \phi_{\text {Mich }}\right|:=\Delta \phi_{u}-\Delta \phi_{v},
\end{gathered}
$$

where $\mathcal{F}$ is the finesse of the Fabry-Perot cavity; $L$ is the arm length of the detector; $\Delta \phi_{u}$ and $\Delta \phi_{v}$ are the phase shifts in the arms $u$ and $v$, respectively; and $f_{p}$ is the pole frequency of the cavity given by

$$
f_{p} \approx \frac{c}{4 \mathcal{F} L} .
$$

To calculate the phase shift of a Michelson interferometer with opening angle $\theta$, we consider an incoming GW with a + polarization:

$$
h_{\mu \nu}^{+}=h_{+}\left(\begin{array}{cccc}
0 & 0 & 0 & 0 \\
0 & 1 & 0 & 0 \\
0 & 0 & -1 & 0 \\
0 & 0 & 0 & 0
\end{array}\right) \cos \left(\omega_{\mathrm{GW}} t\right)
$$

The GW effectively stretches space in the $x$ direction and squeezes it in the $y$ direction, as depicted in Fig. 1, by a factor $h_{+}\left(t-\frac{L}{c}\right)$, using the approximation $\frac{\omega_{\mathrm{GW}} L}{c} \ll 1$. In this choice of reference frame, we can write $\vec{v}$ as

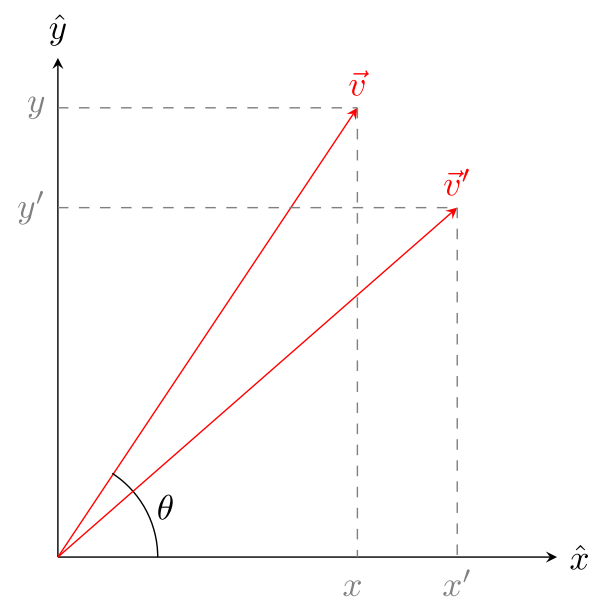

FIG. 1. The detector arm $\vec{v}$ of a detector with opening angle $\theta$ gets deformed to $\vec{v}^{\prime}$ under the influence of a gravitational wave with + polarization. The other detector arm $\vec{u}$ lies on the $x$ axis.

$$
\vec{v}=\left(\begin{array}{l}
x \\
y
\end{array}\right)=L\left(\begin{array}{c}
\cos \theta \\
\sin \theta
\end{array}\right), \quad|\vec{v}|=L
$$

Using the previous approximation, we can write down the components of the deformed arm $\vec{v}^{\prime}$ and express the change in the coordinates as

$$
x^{\prime}=\sqrt{x^{2}+h_{+} x^{2}} \approx\left[1+\frac{1}{2} h_{+}\right] x \Rightarrow \Delta x=\frac{1}{2} h_{+} x
$$

$y^{\prime}=\sqrt{y^{2}-h_{+} y^{2}} \approx\left[1-\frac{1}{2} h_{+}\right] y \Rightarrow \Delta y=-\frac{1}{2} h_{+} y$,

where we used the short notation $h_{+}$for $h_{+}\left(t-\frac{L}{c}\right)$ and expanded to first order. The total change in the length of the detector arm is then given by

$$
\begin{aligned}
\Delta v & =\left|\vec{v}^{\prime}\right|-|\vec{v}|=\sqrt{x^{\prime 2}+y^{\prime 2}}-\sqrt{x^{2}+y^{2}} \\
& =\frac{1}{2} \frac{2 x}{\sqrt{x^{2}+y^{2}}} \Delta x+\frac{1}{2} \frac{2 y}{\sqrt{x^{2}+y^{2}}} \Delta y+\mathcal{O}\left(\Delta^{2}\right) \\
& \approx \frac{\cos \theta L}{L} \Delta x+\frac{\sin \theta L}{L} \Delta y \\
& =\frac{1}{2} h_{+} L\left(\cos ^{2} \theta-\sin ^{2} \theta\right) .
\end{aligned}
$$

Since the light bounces back and forth, the phase shift catches a factor of 2: $\Delta \phi_{u}=2 k_{L} \Delta u$ and $\Delta \phi_{v}=2 k_{L} \Delta v$.

With those results, we can calculate the amplitude of the Michelson phase shift, 


$$
\begin{aligned}
\left|\Delta \phi_{\text {Mich }}\right|= & \left|\Delta \phi_{u}-\Delta \phi_{v}\right| \\
= & \mid k_{L} h_{+}\left(t-\frac{L}{c}\right) L \\
& -k_{L} h_{+}\left(t-\frac{L}{c}\right) L\left(\cos ^{2} \theta-\sin ^{2} \theta\right) \mid \\
= & \frac{4 \pi}{\lambda_{L}} \sin ^{2} \theta L h_{+},
\end{aligned}
$$

with the wave number $k_{L}$ and wave length $\lambda_{L}$ of the laser: $k_{L}=\frac{2 \pi}{\lambda_{L}}$. The change in the path length of a photon due to the incoming $\mathrm{GW}$ is given by

$$
\Delta L=2(\Delta u-\Delta v)=\sin ^{2} \theta L h_{+} .
$$

Therefore, the transfer function (change in path length per GW amplitude) is $\sin ^{2} \theta L$.

By inserting $\left|\Delta \phi_{\text {Mich }}\right|$ and the transfer function for a general opening angle $\theta$ of the detector arms into Eqs. (9.220), (9.234), and (9.122) of Ref. [24] and neglecting the efficiency of the photodetector $\eta \approx 1$, we get the shot noise

$$
\left.\sqrt{S_{n}(f)}\right|_{\text {shot }}=\frac{1}{4 \mathcal{F} \sin ^{2} \theta L} \sqrt{\frac{\pi \hbar \lambda_{L} c}{C P_{0}}} \sqrt{1+\left(\frac{f}{f_{p}}\right)^{2}},
$$

the radiation pressure

$$
\left.\sqrt{S_{n}(f)}\right|_{\mathrm{rad}}=\frac{16 \mathcal{F}}{M \sin ^{2} \theta L} \sqrt{\frac{\hbar C P_{0}}{\pi \lambda_{L} c}} \frac{1}{(2 \pi f)^{2} \sqrt{1+\left(\frac{f}{f_{p}}\right)^{2}}}
$$

and the optical read-out noise thus being given by

$$
\left.S_{n}(f)\right|_{\mathrm{opt}}=\left.S_{n}(f)\right|_{\mathrm{shot}}+\left.S_{n}(f)\right|_{\mathrm{rad}} .
$$

We will use this last result as the main component of the total noise, for an opening angle $\theta(\pi / 3$ for the ET and $\pi / 2$ for LIGO-like detectors).

Each ET detector consists of a high- (HF) and a lowfrequency (LF) detector, which are then used as one to

TABLE I. Detector characteristics of the HF and LF detectors, taken from the Einstein Telescope proposal [14], Sec. 5.1.

\begin{tabular}{lcc}
\hline \hline Quantity & ET-HF & ET-LF \\
\hline $\begin{array}{l}\text { Input power (after input } \\
\text { mode cleaners) } P_{0}\end{array}$ & $500 \mathrm{~W}$ & $3 \mathrm{~W}$ \\
Laser wavelength $\lambda_{L}$ & $1064 \mathrm{~nm}$ & $1550 \mathrm{~nm}$ \\
Arm length $L$ & $10 \mathrm{~km}$ & $10 \mathrm{~km}$ \\
Mirror mass $M$ & $200 \mathrm{~kg}$ & $211 \mathrm{~kg}$ \\
Finesse $\mathcal{F}$ & 880 & 880 \\
Recycling gain $C$ & 21.6 & 21.6 \\
\hline \hline
\end{tabular}

TABLE II. Detector characteristics of the aLIGO detectors, taken from Ref. [25], and $C$ from Ref. [26].

\begin{tabular}{lc}
\hline \hline Quantity & aLIGO \\
\hline Input power (at power recycling mirror) $P_{0}$ & Up to $125 \mathrm{~W}$ \\
Laser wavelength $\lambda_{L}$ & $1064 \mathrm{~nm}$ \\
Arm length $L$ & $4 \mathrm{~km}$ \\
Mirror mass $M$ & $40 \mathrm{~kg}$ \\
Finesse $\mathcal{F}$ & 450 \\
Recycling gain $C$ & 38 \\
\hline \hline
\end{tabular}

broaden the frequency range. The detector characteristics of these two detectors are listed in Table I and will be used throughout this paper. The values we are using for advanced LIGO are summarized in Table II.

\section{Overlap reduction functions $\gamma_{I, J}^{M}(f)$}

The angular dependence of the pattern functions $F_{A}(\hat{\Omega})$ can be split into the relative orientation of the detectors toward each other and the orientation of an incoming GW with respect to the two-detector cluster. The ORFs account for the relative orientation of the two detectors.

We consider a pair $(I, J)$ of Michelson interferometers on Earth with opening angles $\phi_{I}$ and $\phi_{J}$. We denote the direction vectors of the detector arms as $\hat{u}_{I, J}, \hat{v}_{I, J}$ such that $\left(\hat{u}_{I, J}, \hat{v}_{I, J}, \hat{z}_{I, J}\right)$, with $\hat{z}_{I, J}$ being the direction pointing to the sky, forms a positively oriented frame, as shown in Fig. 2. The relative orientation of the detectors can be described by the angles $\sigma_{I, J}$ between the detector arms $\hat{u}_{I, J}$ and the separation vector $\Delta \vec{x}$, which points from detector $I$ to $J$.

The direction vectors of the detector arms in the cluster frame are given by

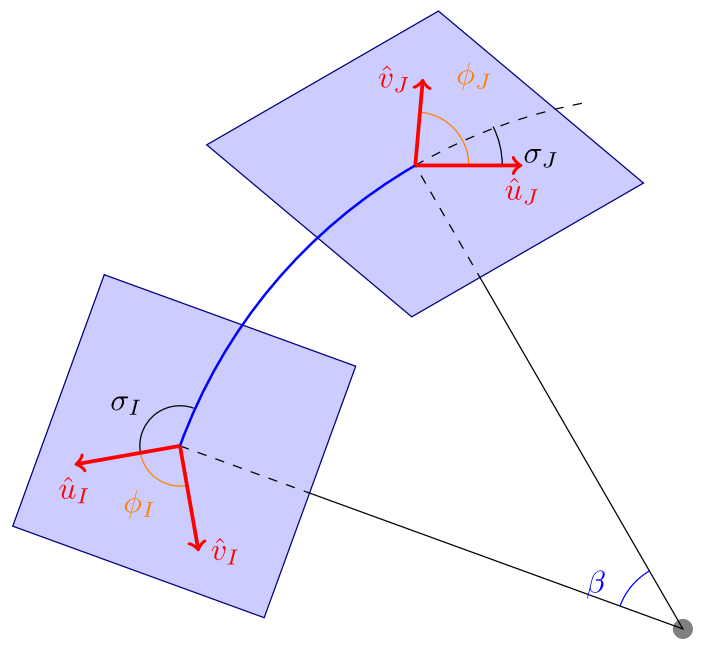

FIG. 2. Depiction of the unit vectors of the detector arms $\hat{u}_{I}, \hat{v}_{I}, \hat{u}_{J}, \hat{v}_{J}$ (red), the opening angles $\phi_{I}, \phi_{J}$ (orange), and the angles $\sigma_{I}$ and $\sigma_{J}$ between $\hat{u}_{I, J}$ and the great circle (blue) between the detectors $I$ and $J$. 


$$
\begin{aligned}
& \hat{u}_{I}=\left(\begin{array}{c}
\cos \sigma_{I} \\
\sin \sigma_{I} \\
0
\end{array}\right), \quad \hat{v}_{I}=\left(\begin{array}{c}
\cos \left(\sigma_{I}+\phi_{I}\right) \\
\sin \left(\sigma_{I}+\phi_{I}\right) \\
0
\end{array}\right), \quad \hat{d}=\frac{1}{\sqrt{2(1-\cos \beta)}}\left(\begin{array}{c}
\sin \beta \\
0 \\
\cos \beta-1
\end{array}\right), \\
& \hat{u}_{J}=\left(\begin{array}{c}
\cos \beta \cos \sigma_{J} \\
\sin \sigma_{J} \\
-\sin \beta \cos \sigma_{J}
\end{array}\right), \quad \hat{v}_{J}=\left(\begin{array}{c}
\cos \beta \cos \left(\sigma_{J}+\phi_{J}\right) \\
\sin \left(\sigma_{J}+\phi_{J}\right) \\
-\sin \beta \cos \left(\sigma_{J}+\phi_{J}\right)
\end{array}\right) .
\end{aligned}
$$

The contractions of the two detector tensors are then given by

$$
\begin{aligned}
D_{I}^{i j} D_{i j}^{J}= & \frac{1}{2}\left(\hat{u}_{I}^{i} \hat{u}_{I}^{j}-\hat{v}_{I}^{i} \hat{v}_{I}^{j}\right) \frac{1}{2}\left(\hat{u}_{i}^{J} \hat{u}_{j}^{J}-\hat{v}_{i}^{J} \hat{v}_{j}^{J}\right) \\
= & \frac{1}{4}\left[\left(\sin ^{2} \sigma_{1+}-\sin ^{2} \sigma_{1-}\right)\left(\sin ^{2} \sigma_{2+}-\sin ^{2} \sigma_{2-}\right)+\frac{1}{2} \cos \beta\left(\sin \left(2 \sigma_{1+}\right)-\sin \left(2 \sigma_{1-}\right)\right)\left(\sin \left(2 \sigma_{2+}\right)-\sin \left(2 \sigma_{2-}\right)\right)\right. \\
& \left.+\cos ^{2} \beta\left(\cos ^{2} \sigma_{1+}-\cos ^{2} \sigma_{1-}\right)\left(\cos ^{2} \sigma_{1+}-\cos ^{2} \sigma_{1-}\right)\right] \\
D_{I, k}^{i} D_{J}^{k j} \hat{d}_{i} \hat{d}_{j}= & \frac{1}{4}\left(\left(\hat{u}_{I} \cdot \hat{d}\right) \hat{u}_{I}-\left(\hat{v}_{I} \cdot \hat{d}\right) \hat{v}_{I}\right) \cdot\left(\left(\hat{u}_{J} \cdot \hat{d}\right) \hat{u}_{J}-\left(\hat{v}_{J} \cdot \hat{d}\right) \hat{v}_{J}\right) \\
= & \frac{1+\cos \beta}{8}\left[\frac{1}{4}\left(\sin \left(2 \sigma_{1+}\right)-\sin \left(2 \sigma_{1-}\right)\right)\left(\sin \left(2 \sigma_{2+}\right)-\sin \left(2 \sigma_{2-}\right)\right)\right. \\
& \left.+\cos \beta\left(\cos ^{2} \sigma_{1+}-\cos ^{2} \sigma_{1-}\right)\left(\cos ^{2} \sigma_{2+}-\cos ^{2} \sigma_{2-}\right)\right] \\
D_{I}^{i j} D_{J}^{k l} \hat{d}_{i} \hat{d}_{j} \hat{d}_{k} \hat{d}_{l}= & \frac{1}{4}\left(\left(\hat{u}_{I} \cdot \hat{d}\right)^{2}-\left(\hat{v}_{I} \cdot \hat{d}\right)^{2}\right)\left(\left(\hat{u}_{J} \cdot \hat{d}\right)^{2}-\left(\hat{v}_{J} \cdot \hat{d}\right)^{2}\right) \\
= & \frac{(1+\cos \beta)^{2}}{16}\left(\cos ^{2} \sigma_{1+}-\cos ^{2} \sigma_{1-}\right)\left(\cos ^{2} \sigma_{2+}-\cos ^{2} \sigma_{2-}\right)
\end{aligned}
$$

with $\sigma_{1+}:=\sigma_{I}+\phi_{I}, \sigma_{1-}:=\sigma_{I}, \sigma_{2+}:=\sigma_{J}+\phi_{J}$, and $\sigma_{2-}:=\sigma_{J}$.

Nishizawa et al. [21] have used a different definition of the angles $\sigma_{1,2}$, which is related to our notation by $\sigma_{1+}=\sigma_{1}+\frac{\phi_{I}}{2}$, $\sigma_{1-}=\sigma_{2}-\frac{\phi_{I}}{2}, \sigma_{2+}=\sigma_{2}+\frac{\phi_{J}}{2}$, and $\sigma_{2-}=\sigma_{2}-\frac{\phi_{J}}{2}$.

Finally, we get the expression for the ORF $\gamma_{I J}^{M}$ of the detectors $I$ and $J$ for the polarization $M$,

$$
\begin{aligned}
\gamma_{I J}^{M}(f)= & \rho_{1}^{M}(\alpha) D_{I}^{i j} D_{i j}^{J}+\rho_{2}^{M}(\alpha) D_{I, k}^{i} D_{J}^{k j} \hat{d}_{i} \hat{d}_{j}+\rho_{3}^{M}(\alpha) D_{I}^{i j} D_{J}^{k l} \hat{d}_{i} \hat{d}_{j} \hat{d}_{k} \hat{d}_{l} \\
= & \frac{1}{16}\left\{4 \rho_{1}^{M}\left(\sin ^{2} \sigma_{1+}-\sin ^{2} \sigma_{1-}\right)\left(\sin ^{2} \sigma_{2+}-\sin ^{2} \sigma_{2-}\right)\right. \\
& +\left(2 \rho_{1}^{M} \cos \beta+\rho_{2}^{M} \frac{1+\cos \beta}{2}\right) \cdot\left(\sin \left(2 \sigma_{1+}\right)-\sin \left(2 \sigma_{1-}\right)\right)\left(\sin \left(2 \sigma_{2+}\right)-\sin \left(2 \sigma_{2-}\right)\right) \\
& \left.\left.+\left(4 \rho_{1}^{M} \cos ^{2} \beta+2 \rho_{2}^{M}(1+\cos \beta) \cos \beta+\rho_{3}^{M}(1+\cos \beta)^{2}\right)\right\} \cdot\left(\cos ^{2} \sigma_{1+}-\cos ^{2} \sigma_{1-}\right)\left(\cos ^{2} \sigma_{2+}-\cos ^{2} \sigma_{2-}\right)\right\},
\end{aligned}
$$

where we defined the argument $\alpha$ and the relation between the arc length $\beta$ and the distance $|\vec{d}|$ by

$$
\alpha(f):=\frac{2 \pi f|\vec{d}|}{c}, \quad|\vec{d}|=2 R_{E} \sin \frac{\beta}{2}
$$




\section{EINSTEIN TELESCOPE AND EARTH-BASED DETECTORS}

As mentioned in the Introduction, the Einstein Telescope is going to be part of the third generation of Earth-based detectors, and we thus want to consider several groundbased networks involving the ET in order to figure out how the ET can affect the overall sensitivity. The estimation of the maximal achievable sensitivity could be of use for future detector designs and expectations in the constraint of cosmological parameters. In particular, we want to investigate the polarizations of the gravitational background and the ET's capability of measuring it.

\section{A. Symmetry of the Einstein Telescope}

Since the ET consists of three detectors, one can form three detector pairs that can be used to cross-correlate the signal. With the resulting three noise-free signals, one could in principle (as we will see below, for the ET, those three signals are not independent) solve for the fraction of the power in each polarization mode (tensor $T$, vector $V$, scalar $S$ ) by using the ORFs.

The fraction in Eq. (18) can be rewritten as

$$
\begin{aligned}
\frac{\operatorname{det} \mathbf{F}}{\mathcal{F}_{T}} & =\frac{\left|\begin{array}{lll}
F_{T T} & F_{T V} & F_{T S} \\
F_{V T} & F_{V V} & F_{V S} \\
F_{S T} & F_{S V} & F_{S S}
\end{array}\right|}{\left|\begin{array}{ll}
F_{V V} & F_{V S} \\
F_{S V} & F_{S S}
\end{array}\right|} \\
& =F_{T T}-\frac{F_{V V} F_{T S}^{2}-2 F_{V S} F_{T S} F_{T V}+F_{S S} F_{T V}^{2}}{F_{V V} F_{S S}-F_{V S}^{2}} .
\end{aligned}
$$

This formula was derived via a maximum likelihood method for more than three detectors to find three modes and is therefore not well defined for two detectors, which can be seen by writing out the expression for $\mathcal{F}_{T}$,

$$
\begin{aligned}
\mathcal{F}_{T} & =\sum_{(I, J)} \sum_{\left(I^{\prime}, J^{\prime}\right)} \int_{0}^{T_{\mathrm{obs}}} \frac{\gamma_{I J}^{V}(t)^{2} \gamma_{I^{\prime} J^{\prime}}^{S}\left(t^{\prime}\right)^{2}-\gamma_{I J}^{V}(t) \gamma_{I J}^{S}(t) \gamma_{I^{\prime} J^{\prime}}^{V}\left(t^{\prime}\right) \gamma_{I^{\prime} J^{\prime}}^{S}\left(t^{\prime}\right)}{P_{I} P_{J} P_{I^{\prime}} P_{J^{\prime}}} d t^{\prime} d t \\
& =\int_{0}^{T_{\mathrm{obs}}} \frac{\gamma_{I J}^{V}(t)^{2} \gamma_{I J}^{S}\left(t^{\prime}\right)^{2}-\gamma_{I J}^{V}(t) \gamma_{I J}^{S}(t) \gamma_{I J}^{V}\left(t^{\prime}\right) \gamma_{I J}^{S}\left(t^{\prime}\right)}{P_{I}^{2} P_{J}^{2}} d t^{\prime} d t=0,
\end{aligned}
$$

where we used that in our case the ORFs are time independent: $\gamma_{I J}^{M}(t)=\gamma_{I J}^{M}(0)$.

Neglecting for the moment factors of $\frac{T_{\mathrm{obs}}}{P_{I} P_{I}}$, we find

$$
\begin{aligned}
\sqrt{F_{V V} F_{S S}} & \sim \sqrt{\sum_{(I, J)} \sum_{\left(I^{\prime}, J^{\prime}\right)}\left(\gamma_{I J}^{V}\right)^{2}\left(\gamma_{I^{\prime} J^{\prime}}^{S}\right)^{2}} \\
& =\sqrt{\left(\gamma_{I J}^{V} \gamma_{I J}^{S}\right)^{2}}=\gamma_{I J}^{V} \gamma_{I J}^{S} \sim F_{V S} \\
\Rightarrow \frac{\operatorname{det} \mathbf{F}}{\mathcal{F}_{T}} & =F_{T T}-\frac{\left(\sqrt{F_{V V}} F_{T S}-\sqrt{F_{S S}} F_{T V}\right)^{2}}{F_{V V} F_{S S}-F_{V S}^{2}} \\
& =\frac{T_{\mathrm{obs}}}{P_{I} P_{J}}\left[\left(\gamma_{I J}^{T}\right)^{2}-\frac{\left(\gamma_{I J}^{V} \gamma_{I J}^{T} \gamma_{I J}^{S}-\gamma_{I J}^{S} \gamma_{I J}^{T} \gamma_{I J}^{V}\right)^{2}}{\left(\gamma_{I J}^{V}\right)^{2}\left(\gamma_{I J}^{S}\right)^{2}-\left(\gamma_{I J}^{V} \gamma_{I J}^{S}\right)^{2}}\right] .
\end{aligned}
$$

This expression is not well defined since the denominator is zero. To see whether the vanishing numerator helps, one has to carefully take the limit of a slightly nondegenerate case. Even if one uses the formula for more than three detectors, one should be careful, since the fraction is ill defined as soon as the $\sum_{(I, J)} \gamma_{I, J}^{M}$ commute, which happens for the three ET detectors due to the fact that they are three identical detectors and their symmetric arrangement leads to $\left[P_{I}=P_{J}=: P\right.$ for every pair $\left.(I, J)\right]$ :

$$
\begin{aligned}
\gamma_{12}^{M} & =\gamma_{23}^{M}=\gamma_{31}^{M} \quad \forall M \in\{T, V, S\} \\
& \Rightarrow F_{M M^{\prime}}=T_{\mathrm{obs}} \frac{\gamma_{M} \gamma_{M^{\prime}}}{P^{2}} \\
& \Rightarrow \sum_{(I, J)}\left(\gamma_{I J}^{M}\right)^{2} \sum_{(I, J)}\left(\gamma_{I J}^{M^{\prime}}\right)^{2}=9\left(\gamma_{I J}^{M}\right)^{2}\left(\gamma_{I J}^{M^{\prime}}\right)^{2} \\
& =\left(\sum_{(I, J)} \gamma_{I J}^{M} \gamma_{I J}^{M^{\prime}}\right)^{2} .
\end{aligned}
$$

To use the formula for the ET detector, we have to break the symmetry by changing the ORF of one detector pair by a small amount $\epsilon(f)$ and then take the limit

$$
\epsilon(f) \rightarrow 0 \quad \forall f .
$$

Without loss of generality, we can, e.g., consider the case $M=T$, and we perturb one of the ORFs:

$$
\gamma_{12}^{M}=\gamma_{23}^{M}=\gamma_{31}^{M}-\epsilon_{M}=: \gamma_{M} \quad \forall M \in\{T, V, S\} .
$$

When we plug this into the denominator and numerator of the fraction in the right-hand side of Eq. (40), we get 


$$
\begin{aligned}
\mathcal{F}_{T} & =F_{V V} F_{S S}-F_{V S}^{2} \\
& =\left(\frac{T_{\mathrm{obs}}}{P^{2}}\right)^{2}\left(\sum_{(I, J)}\left(\gamma_{I J}^{V}\right)^{2} \sum_{(I, J)}\left(\gamma_{I J}^{S}\right)^{2}-\left(\sum_{(I, J)} \gamma_{I J}^{V} \gamma_{I J}^{S}\right)^{2}\right) \\
& =2\left(\frac{T_{\mathrm{obs}}}{P^{2}}\right)^{2}\left(\epsilon_{V} \gamma_{S}-\epsilon_{S} \gamma_{V}\right)^{2}
\end{aligned}
$$

and similarly for the numerator. Plugging these expressions into Eq. (40) and taking the limit, we arrive at

$$
\begin{aligned}
\frac{\operatorname{det} \mathbf{F}}{\mathcal{F}_{T}}= & F_{T T}-\frac{F_{V V} F_{T S}^{2}-2 F_{V S} F_{T S} F_{T V}+F_{S S} F_{T V}^{2}}{F_{V V} F_{S S}-F_{V S}^{2}} \\
= & \frac{T_{\mathrm{obs}}}{P^{2}}\left(\frac{3}{2} \gamma_{T} \epsilon_{T}+\frac{3}{2} \gamma_{V} \gamma_{S} \epsilon_{T} \frac{\epsilon_{T}\left(\gamma_{V} \epsilon_{S}+\gamma_{S} \epsilon_{V}\right)}{\left(\gamma_{V} \epsilon_{S}-\gamma_{S} \epsilon_{V}\right)^{2}}\right. \\
& \left.+3 \gamma_{T} \gamma_{V} \gamma_{S} \epsilon_{T} \frac{\epsilon_{V} \epsilon_{S}}{\left(\gamma_{V} \epsilon_{S}-\gamma_{S} \epsilon_{V}\right)^{2}}\right) \\
& +\mathcal{O}\left(\epsilon^{2}\right) \stackrel{\epsilon \rightarrow 0}{\rightarrow} 0 .
\end{aligned}
$$

Because of the symmetry of the Einstein Telescope, it is thus impossible to separate the modes out of the signal. The ORFs of each detector pair are the same, since they only depend on their relative orientation. Therefore, the detector correlation matrix $\Pi$ has a vanishing determinant, and the relation between the cross-correlated signals and the modes of the gravitational wave background cannot be inverted:

$$
\operatorname{det} \Pi=\left|\begin{array}{lll}
\gamma_{12}^{T} & \gamma_{12}^{V} & \gamma_{12}^{S} \\
\gamma_{23}^{T} & \gamma_{23}^{V} & \gamma_{23}^{S} \\
\gamma_{31}^{T} & \gamma_{31}^{V} & \gamma_{31}^{S}
\end{array}\right|=\left|\begin{array}{lll}
\gamma_{T} & \gamma_{V} & \gamma_{S} \\
\gamma_{T} & \gamma_{V} & \gamma_{S} \\
\gamma_{T} & \gamma_{V} & \gamma_{S}
\end{array}\right|=0
$$

Note that, even by perturbing the symmetry of the ET (slightly changing the arm length or tilting the detector plane), the induced changes are negligible and do not allow the use of the ET alone to distinguish between the polarization modes. A detailed calculation of the symmetry breakings can be found in Appendix A 1.

\section{B. Cross-correlation of future Earth-based detectors}

We now turn our attention to combinations of the ET with other detectors, which are already existing (LIGO and Virgo) or under construction (KAGRA). By adding two additional signals to the ET cluster, we break the symmetry, and the problem mentioned in the previous section is solved. We add the two advanced LIGO detectors in Livingston (LL) and Hanford (LH) to our set of detectors, which results in six correlation signals, out of which four (ET-ET, ET-LL, ET-LH, LL-LH) are independent. This allows us to distinguish the polarization modes, even if one of the detectors could not be used for some reason.

In Fig. 3, we compare the noise power spectral densities of the ET, LIGO, Virgo, and KAGRA and show their combined sensitivity for the polarization modes of a GW signal.
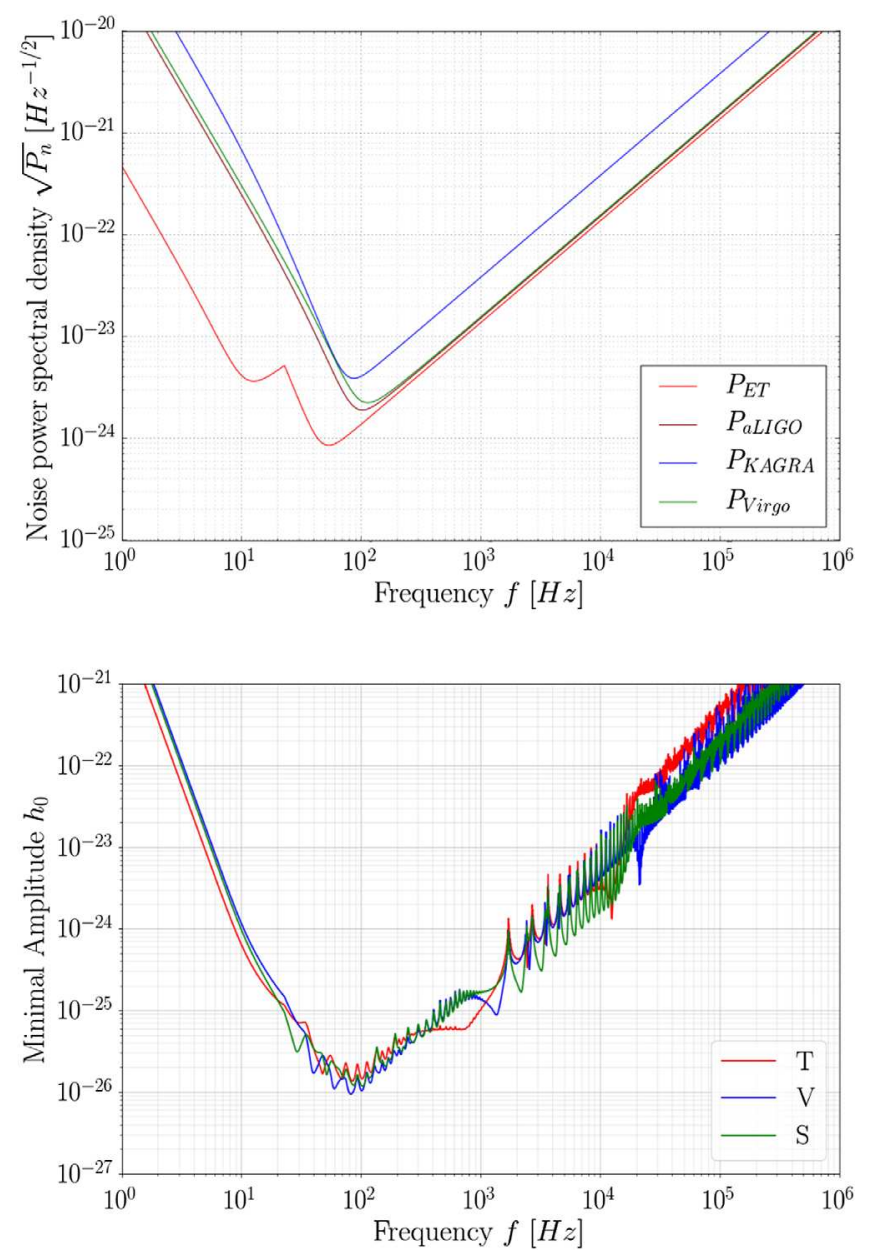

FIG. 3. The noise power spectral densities of all involved detectors (above) and the sensitivity of all existing and nearfuture Earth detectors combined (ET, LIGO, Virgo, and KAGRA). The minimal achievable sensitivity is considered for a SNR of 8 .

Note that all ET detectors lie in the same plane and are on the scale of Earth at the same position. Should one of the three ET detectors be taken out of the network for any reason, the directions in which its arms were pointing would still be covered by the neighboring detectors. This is why the sensitivity would not be significantly affected.

Moreover, by considering the addition of Virgo and KAGRA to the network, besides the ET and LIGO, we slightly gain sensitivity for frequencies above $100 \mathrm{~Hz}$.

\section{DECIGO AND CORRELATION WITH EARTH DETECTORS}

After having considered the combined sensitivity of a strictly Earth-based network of detectors, we can now investigate the consequences of a future space-borne detector. As already mentioned in the Introduction, we focus on the DECIGO project, since the LISA sensitivity lies in a lower frequency range than the Earth detectors and 
no overlap of their respective frequency bands would be possible.

\section{A. Earth-space network sensitivity}

DECIGO is a space-based experiment, and therefore there is no noise due to vibrations of the ground. Since its sensitive region and the one of the ET and LIGO overlap in the frequency range between 10 and $100 \mathrm{~Hz}$, it makes sense to cross-correlate their signal to get a higher precision and confidence for the separation of the signal into the three different polarization modes.

The DECIGO experiment consists of four detector clusters. Each cluster is made up of three satellites, which form three independent identical Michelson interferometers. One can, e.g., arrange the four clusters in the C3 configuration $[17,18]$, in which two clusters are located at the same position near the Earth (about 1 AU (astronomical unit) behind the Earth, on the same orbit around the Sun) and form a star shape and the remaining two form a triangle together with the star cluster, which has the Sun at its center. In Fig. 4, we compare the noise power spectrum of
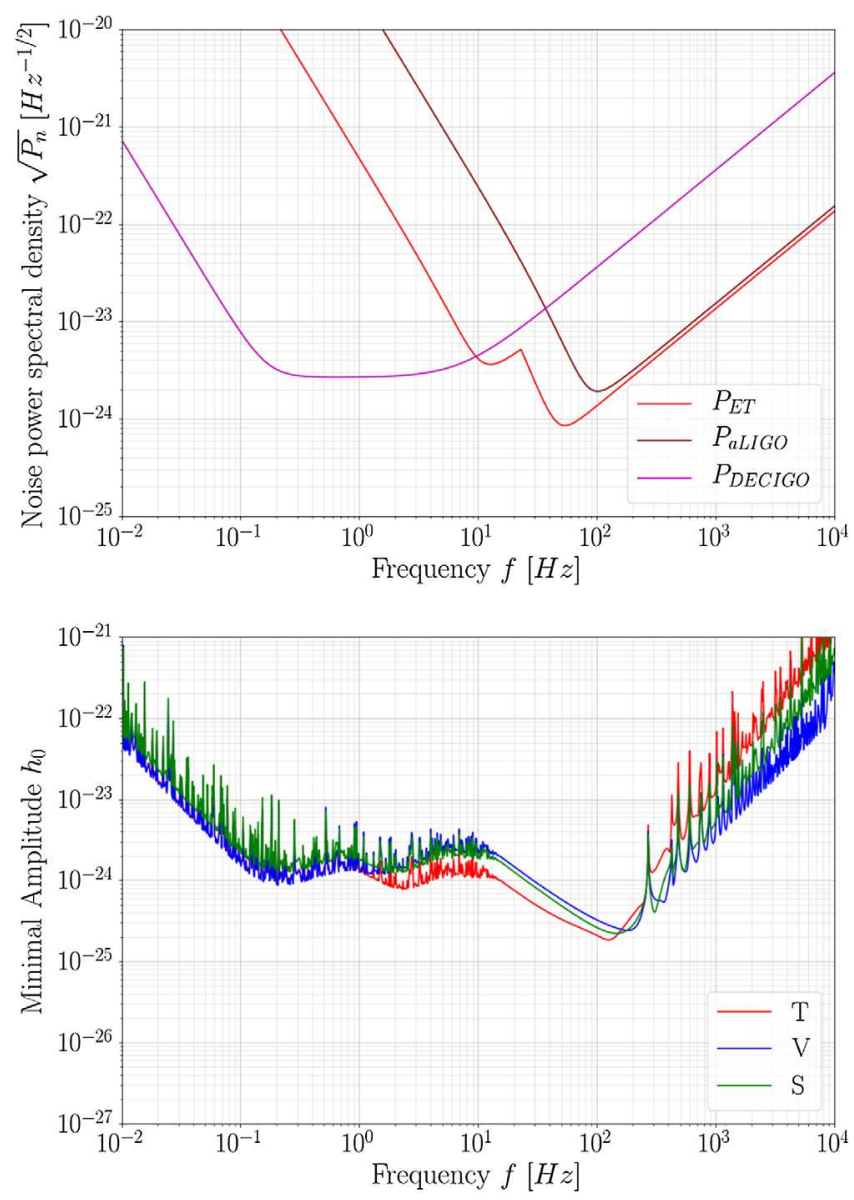

FIG. 4. Noise power spectra of a DECIGO, ET, and advanced LIGO detector (above) and the sensitivity of DECIGO alone in its C3 configuration.
DECIGO to the ones of the ET and LIGO and plot the sensitivity of DECIGO in the $\mathrm{C} 3$ configuration.

DECIGO is much more sensitive in the low frequencies than all detectors on Earth combined and is even slightly more sensitive around $10 \mathrm{~Hz}$, which comes in handy when we combine it with Earth detectors. When we add the ET and then LIGO to the set of detectors and sum over all combinations of cross-correlations, we get the plots shown in Fig. 5.

As we can see, the ET drags the curves down around $10 \mathrm{~Hz}$ and mostly above $100 \mathrm{~Hz}$. In particular, the tensor and scalar modes are affected and become about as sensitive as the vector mode. Together with LIGO, the sensitivity is enhanced by 1 order of magnitude at LIGO's most sensitive frequency range around $100 \mathrm{~Hz}$.

\section{B. Time-dependent sensitivity}

In the planned $\mathrm{C} 3$ configuration of DECIGO we used previously, each cluster rotates around its own axis
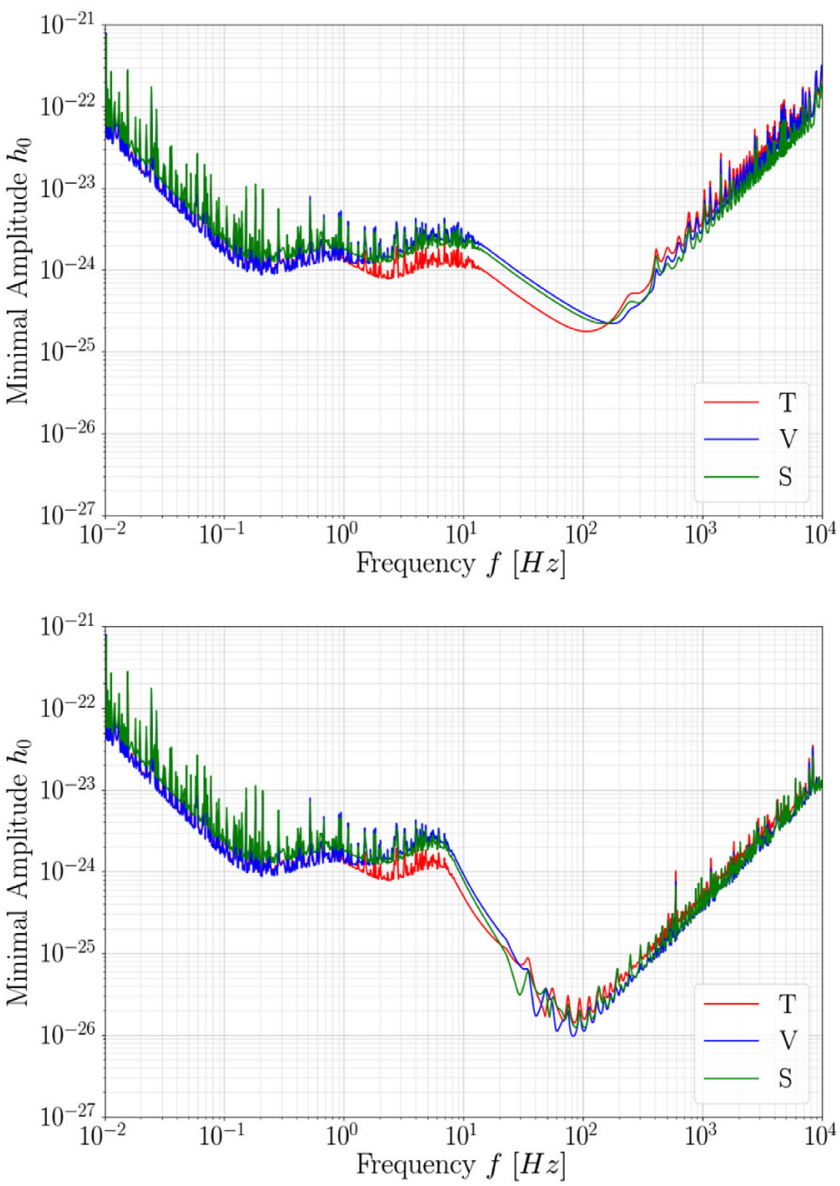

FIG. 5. The ET and all DECIGO detectors in the C3 configuration (above) and together with both LIGO detectors (below), averaged over a total measurement time of one year. The addition of the LIGO detectors significantly improves the sensitivity around $100 \mathrm{~Hz}$. Virgo and KAGRA were not included, since the effect is negligible. 
perpendicular to the detector plane as it rotates around the Sun, such that it returns to its original position after one year. A detector on Earth follows Earth rotation and therefore a relatively quick oscillation of one day superposed to a slow oscillation of one year. This combined change in the orientations of the detectors in a DECIGO cluster relative to detectors on Earth leads to a time-varying sensitivity, which is different for each mode, as can be seen in Fig. 6. The time dependence of the sensitivity is independent of the frequency. We plot the sensitivities at $100 \mathrm{~Hz}$, where the DECIGO-Earth detector pairs are most sensitive.

The variation of the sensitivity with time is different if we form the pair with a DECIGO detector close to the Earth or one far away from it. The location of the peaks is also different for DECIGO-Earth pairs, formed with different DECIGO detectors. Changing the detector on Earth, however, does not matter, since they are almost at the same place viewed on the solar system scale and oscillate much faster and therefore do not influence the trend on a monthly scale.

We now optimize the sensitivity by combining all detector pairs with a similar time dependence and average
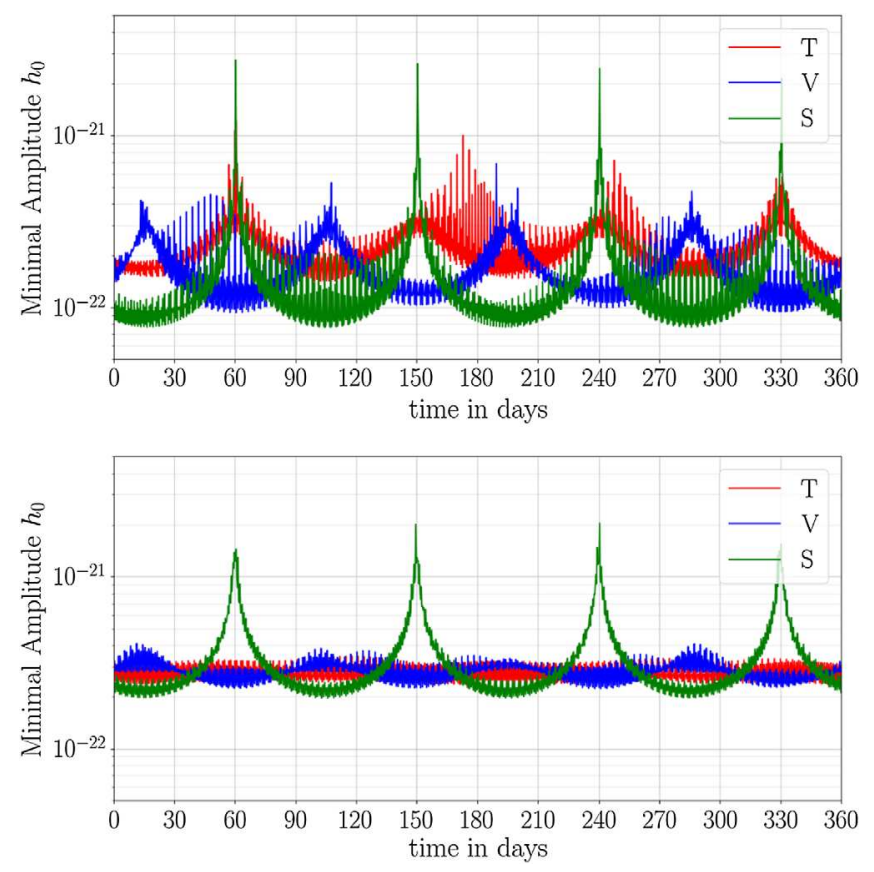

FIG. 6. Above: There are two DECIGO detectors in the star cluster, which show the same time dependence when crosscorrelated with an Earth detector. We take all correlations between those two and all ET and LIGO detectors. Below: In a square configuration, in which the four DECIGO clusters are located on each corner of a square placed around the Sun, one corner is close to the Earth whereas the three other ones are far away from it. These three distant clusters have the same time dependence when we correlate them with Earth detectors. We form all pairs of these three with the ET and LIGO. Both plots correspond to a frequency of $100 \mathrm{~Hz}$. over an integration time of five days in order to not lose to much of the variation. For this purpose, we can always form all pairs with the Earth detectors, but we have to be careful which space detectors we pick. If we want to be able to clearly separate the vector mode from the other two, then it makes sense to pick the $\mathrm{C} 3$ configuration because it has many detectors close to Earth. For two of the detectors in the star cluster, the correlation with any Earth detector has almost the same time dependence since their orientation only differs by $30^{\circ}$. In Fig. 7, we use all those pairs and integrate over five days to increase the sensitivity. Because the vector modes time dependence is phase shifted with respect to the other two modes, we can easily separate it from the other two in this case.

However, if we rather want to identify the scalar mode, then it makes more sense to move more clusters farther away from Earth and at best on the opposite side of the orbit around the Sun, because for detectors that are far away from Earth, the scalar mode has large peaks that correspond to blind spots. In this case, we could arrange the four DECIGO clusters in a square around the Sun, such that only one cluster is close to Earth and one is on the opposite side of Earth orbit. We can then arrange the initial orientation of the clusters, such that the peaks for one detector of each of the three clusters far from Earth coincide. Their combined time-dependent sensitivity is shown in Fig. 8.

By using the same detectors as previously and combining the data differently, one gets an alternative method to the maximum likelihood method for distinguishing the polarization modes. This can help in checking the results and gives a higher confidence on a test of GR, without having to build another experiment.

Note that we performed the same analysis for a scaleddown version of DECIGO closer to the Earth, B-DECIGO [19]. Although the sensitivity curves are a bit similar to the DECIGO ones, the results of the time-dependent sensitivities do not appear to provide good enough differences between the modes. All the details regarding B-DECIGO can be found in Appendix B.

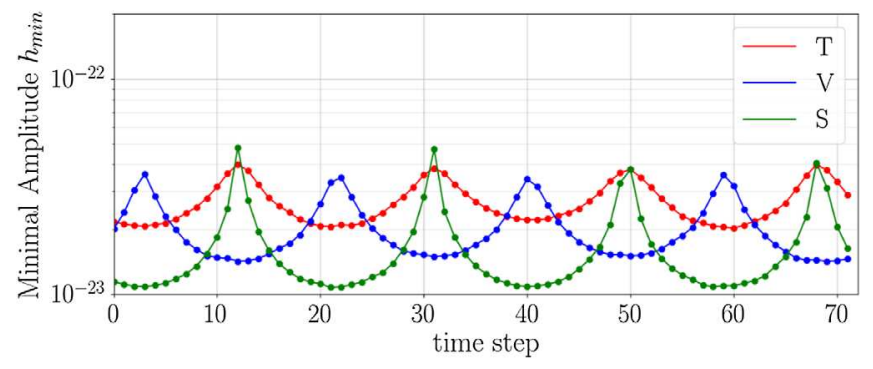

FIG. 7. Combined sensitivity of the detector pairs with one of two neighboring detectors in the star cluster and all ground-based detectors with an integration time of five days at a frequency of $100 \mathrm{~Hz}$ for one year. 


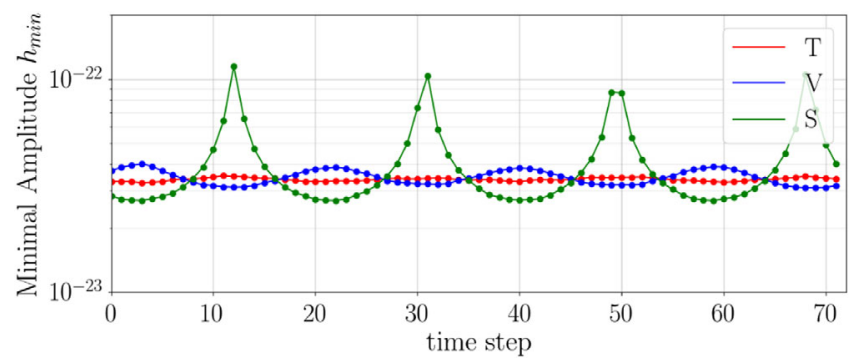

FIG. 8. Time-dependent sensitivity of detector pairs with one of each of the three clusters far from Earth in the square configuration with all Earth detectors, binned in time with steps of five days at a frequency of $100 \mathrm{~Hz}$ for one year.

\section{GRAVITATIONAL WAVES FROM POINT SOURCES}

Until now, we have calculated the sensitivity of various combinations of $\mathrm{GW}$ detectors to an isotropic gravitational wave background. Now, we attempt to do the same for point sources. Since the signal of a point source is coming from a specific direction, we do not average over all solid angles, and our sensitivity becomes direction dependent.

\section{A. Sensitivity}

The derivation of the expression for the signal-tonoise ratio works in analogy to what we have done in Sec. II. The metric perturbation field at the location of the detector $\vec{x}_{I}$ can be described as the sum of all gravitational waves, incident on the detector $I$, coming from all directions:

$$
\begin{aligned}
h_{i j}\left(t, \vec{x}_{I}\right) & =\sum_{A} \int_{\mathbb{S}^{2}} h_{A}\left(t, \vec{x}_{I}, \hat{\Omega}\right) e_{i j}^{A}(\hat{\Omega}) d \hat{\Omega} \\
& =\sum_{A} \iint_{\mathbb{S}^{2}} \tilde{h}_{A}(f, \hat{\Omega}) e^{2 \pi i f\left(t-\frac{\hat{\Omega} \cdot \vec{x}}{c}\right)} e_{i j}^{A}(\hat{\Omega}) d \hat{\Omega} d f .
\end{aligned}
$$

For a gravitational wave coming from a point source located at $\hat{\Omega}_{0}$ in the sky, the frequency-space amplitude takes the form

$$
\tilde{h}_{A}(f, \hat{\Omega})=\hat{h}_{A}(f) \delta\left(\hat{\Omega}-\hat{\Omega}_{0}\right) .
$$

The response of the detector $I$ to an incoming gravitational wave is described by the so-called pattern functions $F_{I}^{A}$, which are defined by contracting the basis tensors $e^{A}$ of the metric perturbations due to GWs for the polarizations $A \in\{+, \times, x, y, b, l\}$ with the detector tensor $D_{I}$ :

$$
F_{I}^{A}(\hat{\Omega}):=e_{i j}^{A}(\hat{\Omega}) D_{I}^{i j} .
$$

Therefore, the Fourier transform of the signal is given by

$$
\tilde{h}_{I}(f)=\sum_{A} \tilde{h}_{A}(f) e^{-2 \pi i f \frac{\hat{\Omega}_{0} \cdot \vec{x}_{I}}{c}} F_{I}^{A}\left(\hat{\Omega}_{0}\right) .
$$

By cross-correlating two strains of different detectors $\left(s_{I}\right.$, $s_{J}$ ), we get rid of the noise as seen in Eq. (12). Thus, the expectation of the Fourier transform of the two strains is

$$
\begin{aligned}
\mathbb{E}\left[\tilde{h}_{I}^{*}(f) \tilde{h}_{J}\left(f^{\prime}\right)\right]= & \tilde{h}_{A}^{*}(f) \tilde{h}_{A^{\prime}}\left(f^{\prime}\right) e^{-\frac{2 \pi i}{c} \hat{\Omega}_{0} \cdot\left(f \vec{x}_{I}-f^{\prime} \vec{x}_{J}\right)} \\
& \cdot F_{I}^{A}\left(\hat{\Omega}_{0}\right) F_{J}^{A^{\prime}}\left(\hat{\Omega}_{0}\right) .
\end{aligned}
$$

To maximize the signal-to-noise ratio, we filter this cross-correlated strain with a filter function $\tilde{Q}$,

$$
\begin{aligned}
\mu:=\mathbb{E}[Y]= & \int \delta_{T}\left(f-f^{\prime}\right) \mathbb{E}\left[\tilde{h}_{I}^{*}(f) \tilde{h}_{J}\left(f^{\prime}\right)\right] \tilde{Q}\left(f^{\prime}\right) d f^{\prime} d f \\
= & \int \tilde{h}_{A}^{*}(f) \tilde{h}_{A^{\prime}}(f) e^{-\frac{2 \pi i f}{c} \hat{\Omega}_{0} \cdot\left(\vec{x}_{I}-\vec{x}_{J}\right)} \\
& \cdot F_{I}^{A}\left(\hat{\Omega}_{0}\right) F_{J}^{A^{\prime}}\left(\hat{\Omega}_{0}\right) \tilde{Q}(f) d f,
\end{aligned}
$$

where $Y$ is the cross-correlated signal:

$Y:=\int_{-\infty}^{\infty} \int_{-\infty}^{\infty} \delta_{T}\left(f-f^{\prime}\right) \tilde{s}_{I}^{*}(f) \tilde{s}_{J}\left(f^{\prime}\right) \tilde{Q}\left(f-f^{\prime}\right) d f^{\prime} d f$.

To find the optimal filter function $\tilde{Q}$, we define a scalar product on the space of smooth complex valued functions $C^{\infty}(\mathbb{C})$ :

$$
(A, B):=\int A^{*}(f) B(f) P_{I}(|f|) P_{J}(|f|) d f .
$$

Since the noise power spectra diverge algebraically at the origin and at infinity, we have to restrict our functions $A$ and $B$ to the Schwartz space $\mathcal{S}(\mathbb{C})$.

We can express the expectation of the correlated signal and its variance in terms of this scalar product,

$$
\begin{aligned}
\mu & =\left(\tilde{Q}, \frac{\tilde{h}_{A}^{*} \tilde{h}_{A^{\prime}} e^{-\frac{2 \pi i f}{c} \hat{\Omega}_{0} \cdot \Delta \vec{x}} F_{I}^{A} F_{J}^{A^{\prime}}}{P_{I} P_{J}}\right), \\
\sigma^{2} & :=\mathbb{V}[Y]=\mathbb{E}\left[Y^{2}\right]-\mathbb{E}[Y]^{2} \approx \mathbb{E}\left[Y^{2}\right]=\frac{T}{4}(\tilde{Q}, \tilde{Q}),
\end{aligned}
$$

where $\Delta \vec{x}:=\vec{x}_{I}-\vec{x}_{J}$ is the distance vector between the detectors $I$ and $J$. 
The signal-to-noise ratio is therefore given by

$$
\mathrm{SNR}=\frac{\mu}{\sigma}=\frac{\left(\tilde{Q}, \frac{\tilde{h}_{A}^{*} \tilde{h}_{A^{\prime}} e^{-\frac{2 \pi i f}{c} \hat{\Omega}_{0} \cdot \Delta \vec{x}} F_{I}^{A} F_{J}^{A^{\prime}}}{P_{I} P_{J}}\right)}{\sqrt{\frac{T}{4}(\tilde{Q}, \tilde{Q})}} .
$$

This can be maximized by choosing the filter function $\tilde{Q}$ parallel to the correlated signal with respect to our scalar product,

$$
\tilde{Q} \propto \frac{\tilde{h}_{A}^{*} \tilde{h}_{A^{\prime}} e^{-\frac{2 \pi i f}{c} \hat{\Omega}_{0} \cdot \Delta \vec{x}} F_{I}^{A} F_{J}^{A^{\prime}}}{P_{I} P_{J}}=:\left\langle h_{I} h_{J}\right\rangle .
$$

With a proportionality constant $K$, we get

$$
\begin{aligned}
\mathrm{SNR} & =\sqrt{\frac{4}{T}} \frac{\left(K\left\langle h_{I} h_{J}\right\rangle,\left\langle h_{I} h_{J}\right\rangle\right)}{\sqrt{\left(K\left\langle h_{I} h_{J}\right\rangle, K\left\langle h_{I} h_{J}\right\rangle\right)}} \\
& =2 \sqrt{T\left(\left\langle h_{I} h_{J}\right\rangle,\left\langle h_{I} h_{J}\right\rangle\right)} .
\end{aligned}
$$

Without loss of generality, we can therefore choose $\tilde{Q}=\left\langle h_{I} h_{J}\right\rangle$. Finally, we can calculate the maximal possible signal-to-noise ratio, with this choice of optimal filter function,

$$
\begin{aligned}
\mathrm{SNR} & =2 \sqrt{\frac{1}{T}(\tilde{Q}, \tilde{Q})} \\
& =2 \sqrt{\frac{1}{T} \int \frac{\left(\tilde{h}_{A}(f) \tilde{h}_{A^{\prime}}(f) F_{I}^{A}\left(\hat{\Omega}_{0}\right) F_{J}^{A^{\prime}}\left(\hat{\Omega}_{0}\right)\right)^{2}}{P_{I}(|f|) P_{J}(|f|)} d f .}
\end{aligned}
$$

To extract the frequency dependence and the polarization, we insert a harmonic wave with amplitude $h_{0}$, frequency $f$, and polarization $A$ :

$$
\begin{aligned}
& \tilde{h}(f) \stackrel{!}{=} h_{0} \delta_{T}\left(f-f_{0}\right) \delta_{A^{\prime} A} ; \\
& \mathrm{SNR}=2 \sqrt{T^{2} \frac{\left(\left|h_{0}\right|^{2} F_{I}^{A}\left(\hat{\Omega}_{0}\right) F_{J}^{A} \hat{\Omega}_{0}\right)^{2}}{P_{I}(|f|) P_{J}(|f|)}} \\
&=2 T \frac{\left|h_{0}\right|^{2} F_{I}^{A}\left(\hat{\Omega}_{0}\right) F_{J}^{A}\left(\hat{\Omega}_{0}\right)}{\sqrt{P_{I}(|f|) P_{J}(|f|)}} .
\end{aligned}
$$

So, we get the minimal amplitude required to detect a gravitational wave with polarization $A$ and at a SNR of at least 8:

$$
\left|h_{0}^{A}(f)\right|_{\min }=\sqrt{32} \frac{\sqrt[4]{P_{I}(|f|) P_{J}(|f|)}}{\sqrt{T F_{I}^{A}\left(\hat{\Omega}_{0}\right) F_{J}^{A}\left(\hat{\Omega}_{0}\right)}} .
$$

\section{B. Determination of location and polarizations of point sources}

If one has more than eight detector pairs $(I, J)$ (DECIGO would do, e.g.), one can solve for the direction $\hat{\Omega} \simeq(\theta, \phi)$ and all six possible polarizations $A \in\{+, \times, x, y, b, l\}$ of an incoming gravitational wave from a point source. We determine the SNR for each quantity under the assumption that the maximum likelihood method is used to calculate them from the at least eight cross-correlated signals $\mu_{I J}$. The derivation is analogous to the one given by Nishizawa et al. [22].

The true parameters are denoted by $\vec{\theta}_{\text {true }}=\left(Y, s_{A}, \hat{\omega}\right)$ :

$Y_{I J}(f)=T^{3 / 2}\left|\sum_{A} \tilde{s}_{A}(f) F_{I}^{A}(\hat{\omega}) \sum_{A^{\prime}} \tilde{s}_{A^{\prime}}(f) F_{J}^{A^{\prime}}(\hat{\omega})\right|$.

The estimated values are $\mu=\langle Y\rangle, h_{A}=\left\langle s_{A}\right\rangle, \hat{\Omega}=\langle\hat{\omega}\rangle$,

$$
\begin{aligned}
\mu_{I J}(f) & =Y_{I J}(f)+n_{I J}(f) \\
& =T^{3 / 2}\left|\sum_{A} \tilde{h}_{A}(f) F_{I}^{A}(\hat{\Omega}) \sum_{A^{\prime}} \tilde{h}_{A^{\prime}}(f) F_{J}^{A^{\prime}}(\hat{\Omega})\right|,
\end{aligned}
$$

where the noise $n_{I J}(f)$ satisfies

$$
\begin{aligned}
\mathbb{E}\left[n_{I J}(f)\right] & =0, \\
\mathbb{V}\left[n_{I J}(f)\right] & =\frac{T}{4} P_{I}(f) P_{J}(f)=: \mathcal{N}_{I J}(f) .
\end{aligned}
$$

Our likelihood function is given by

$$
L\left(\mu_{I J} \mid \vec{\theta}\right)=\exp \left[-\sum_{(I, J)} \frac{\left(Y_{I J}-\mu_{I J}\right)^{2}}{2 \mathcal{N}_{I J}}\right]
$$

with the parameters $\vec{\theta}=(\theta, \phi,+, \times, x, y, b, l)$.

The Fisher information matrix can then be calculated as follows:

$$
\begin{aligned}
F_{i j} & =\mathbb{E}\left[\left(\partial_{\theta_{i}} \ln L\left(\mu_{I J} \mid \vec{\theta}\right)\right)\left(\partial_{\theta_{j}} \ln L\left(\mu_{I J} \mid \vec{\theta}\right)\right)\right], \\
\mathbf{F} & =\left(\begin{array}{lll}
F_{\theta \theta} & F_{\theta \phi} & F_{\theta A^{\prime}} \\
F_{\phi \theta} & F_{\phi \phi} & F_{\phi A^{\prime}} \\
F_{A \theta} & F_{A \phi} & F_{A A^{\prime}}
\end{array}\right) .
\end{aligned}
$$

To simplify the notation, we define $\alpha^{\prime}:=\frac{2 \pi f}{c}$. We now calculate the $\theta \theta$ and the $\theta A$ components for a $\mathrm{GW}$ with polarization $A_{0}$ : 


$$
\begin{aligned}
& \left.F_{\theta \theta}\right|_{h=h_{A_{0}}}=\left.\mathbb{E}\left[\left(\partial_{\theta} \ln L\right)^{2}\right]\right|_{h=h_{A_{0}}}=\mathbb{E}\left[\left(\sum_{(I, J)} \frac{1}{\mathcal{N}_{I J}}\left(Y_{I J}-\mu_{I J}\right) T^{3}\left|\tilde{h}_{A_{0}}\right|^{2} \partial_{\theta} F_{I}^{A_{0}} F_{J}^{A_{0}}\right)^{2}\right] \\
& =\sum_{(I, J)} \frac{1}{\mathcal{N}_{I J}^{2}} \underbrace{\mathbb{E}\left[\left(Y_{I J}-\mu_{I J}\right)^{2}\right]}_{=\mathcal{N}_{I J}} T^{3}\left(\left|\tilde{h}_{A_{0}}\right|^{2} \partial_{\theta} F_{I}^{A_{0}} F_{J}^{A_{0}}\right)^{2} \\
& +\sum_{(I, J) \neq\left(I^{\prime}, J^{\prime}\right)} \frac{1}{\mathcal{N}_{I J} \mathcal{N}_{I^{\prime} J^{\prime}}} \underbrace{\mathbb{E}\left[\left(Y_{I J}-\mu_{I J}\right)\left(Y_{I^{\prime} J^{\prime}}-\mu_{I^{\prime} J^{\prime}}\right)\right]}_{=0} T^{3}\left(\left|\tilde{h}_{A_{0}}\right|^{2} \partial_{\theta} F_{I}^{A_{0}} F_{J}^{A_{0}}\right)\left(\left|\tilde{h}_{A_{0}}\right|^{2} \partial_{\theta} F_{I^{\prime}}^{A_{0}} F_{J^{\prime}}^{A_{0}}\right) \\
& =\sum_{(I, J)} \frac{T^{3}}{\mathcal{N}_{I J}}\left(\left|\tilde{h}_{A_{0}}\right|^{2} \partial_{\theta} F_{I}^{A_{0}} F_{J}^{A_{0}}\right)^{2},
\end{aligned}
$$

$$
\begin{aligned}
\left.F_{\theta A}\right|_{h=h_{A_{0}}} & =\left.\mathbb{E}\left[\left(\partial_{\theta} \ln L\right)\left(\partial_{\left|\tilde{h}_{A}\right|^{2}} \ln L\right)\right]\right|_{h=h_{A_{0}}} \\
& =\mathbb{E}\left[\left.\left(\sum_{(I, J)} \frac{1}{\mathcal{N}_{I J}}\left(Y_{I J}-\mu_{I J}\right) T^{\frac{3}{2}}\left|\tilde{h}_{A_{0}}\right|^{2} \partial_{\theta} F_{I}^{A_{0}} F_{J}^{A_{0}}\right)\left(\sum_{(I, J)} \frac{1}{\mathcal{N}_{I J}}\left(Y_{I J}-\mu_{I J}\right) T^{\frac{3}{2}} \partial_{\left|h_{A}\right|^{2}}\left|\tilde{h}_{I} \tilde{h}_{J}\right|\right)\right|_{h=h_{A_{0}}}\right] \\
& =\sum_{(I, J)} \frac{T^{3}}{\mathcal{N}_{I J}}\left(F_{I}^{A} F_{J}^{A}+\left(1-\delta_{A A_{0}}\right) \frac{1}{2}\left[\frac{\left(F_{I}^{A}\right)^{2} F_{J}^{A_{0}}}{F_{I}^{A_{0}}}+\frac{\left(F_{J}^{A}\right)^{2} F_{I} A_{0}}{F_{J}^{A_{0}}}\right]\right)\left|\tilde{h}_{A_{0}}\right|^{2} \partial_{\theta} F_{I}^{A_{0}} F_{J}^{A_{0}} .
\end{aligned}
$$

A detailed calculation of the matrix elements for the more general case, in which we have different integration times for different detectors, can be found in Appendix D. We list here the rest of the components again for an $A_{0}$ polarized wave:

$$
\begin{aligned}
F_{\phi \phi} & =\sum_{(I, J)} \frac{T^{3}}{\mathcal{N}_{I J}}\left(\left|\tilde{h}_{A_{0}}\right|^{2} \partial_{\phi} F_{I}^{A_{0}} F_{J}^{A_{0}}\right)^{2}, \\
F_{\theta \phi} & =\sum_{(I, J)} \frac{T^{3}}{\mathcal{N}_{I J}}\left(\left|\tilde{h}_{A_{0}}\right|^{2}\right)^{2}\left(\partial_{\theta} F_{I}^{A_{0}} F_{J}^{A_{0}}\right)\left(\partial_{\phi} F_{I}^{A_{0}} F_{J}^{A_{0}}\right) \\
F_{\phi A} & =\sum_{(I, J)} \frac{T^{3}}{\mathcal{N}_{I J}}\left(F_{I}^{A} F_{J}^{A}+\left(1-\delta_{A A_{0}}\right) \frac{1}{2}\left[\frac{\left(F_{I}^{A}\right)^{2} F_{J}^{A_{0}}}{F_{I}^{A_{0}}}+\frac{\left(F_{J}^{A}\right)^{2} F_{I}^{A_{0}}}{F_{J}^{A_{0}}}\right]\right)\left|\tilde{h}_{A_{0}}\right|^{2} \partial_{\phi} F_{I}^{A_{0}} F_{J}^{A_{0}}, \\
F_{A A^{\prime}} & =\sum_{(I, J)} \frac{T^{3}}{\mathcal{N}_{I J}}\left(F_{I}^{A} F_{J}^{A}+\left(1-\delta_{A A_{0}}\right) \frac{1}{2}\left[\frac{\left(F_{I}^{A}\right)^{2} F_{J}^{A_{0}}}{F_{I}^{A_{0}}}+\frac{\left(F_{J}^{A}\right)^{2} F_{I}^{A_{0}}}{F_{J}^{A_{0}}}\right]\right) \cdot\left(F_{I}^{A^{\prime}} F_{J}^{A^{\prime}}+\left(1-\delta_{A^{\prime} A_{0}}\right) \frac{1}{2}\left[\frac{\left(F_{I}^{A^{\prime}}\right)^{2} F_{J}^{A_{0}}}{F_{I}^{A_{0}}}+\frac{\left(F_{J}^{A^{\prime}}\right)^{2} F_{I}^{A_{0}}}{F_{J}^{A_{0}}}\right]\right) .
\end{aligned}
$$

The inverse of the Fisher matrix is the covariance matrix, which has the variance of $\theta_{i}$ in the $i$ th diagonal entry. So, the square of the SNR for measuring polarization $A$ is given by

$$
\operatorname{SNR}_{A}^{2}:=\left.\frac{\left(\left|\tilde{h}_{A}\right|^{2}\right)^{2}}{\sigma_{A}^{2}}\right|_{h=h_{A}}=\left.\frac{\left(\left|\tilde{h}_{A}\right|^{2}\right)^{2}}{\left(\mathbf{F}^{-1}\right)_{A A}}\right|_{h=h_{A}}=\left.\frac{\left(\left|\tilde{h}_{A}\right|^{2}\right)^{2} \operatorname{det} \mathbf{F}}{\mathcal{F}_{A}}\right|_{h=h_{A}},
$$

where $\mathcal{F}_{\theta_{i}}$ is the determinant of the minor one gets from removing the $i$ th row and column from the Fisher matrix $\mathbf{F}$. The SNR of the cross-correlation is related to the one of amplitude by

$$
\operatorname{SNR}[\mu]=\operatorname{SNR}\left[h^{2}\right]=\operatorname{SNR}^{2}[h] .
$$

Again, demanding an $\operatorname{SNR}[h]$ of at least 8 gives us the minimal amplitude. We can read off the prefactors by comparing with the result for one detector pair above:

$$
\left|\tilde{h}_{A}\right|_{\min }=\sqrt{\frac{32}{T}} \sqrt[4]{\left|\frac{\mathcal{F}_{A}}{\operatorname{det} \mathbf{F}}\right|_{h=h_{A}}} .
$$

The variance of the position in the sky is given by

$$
\begin{gathered}
\mathbb{V}[\theta]=\left(\mathbf{F}^{-1}\right)_{\theta \theta}=\frac{\mathcal{F}_{\theta}}{\operatorname{det} \mathbf{F}}, \\
\mathbb{V}[\phi]=\left(\mathbf{F}^{-1}\right)_{\phi \phi}=\frac{\mathcal{F}_{\phi}}{\operatorname{det} \mathbf{F}} .
\end{gathered}
$$




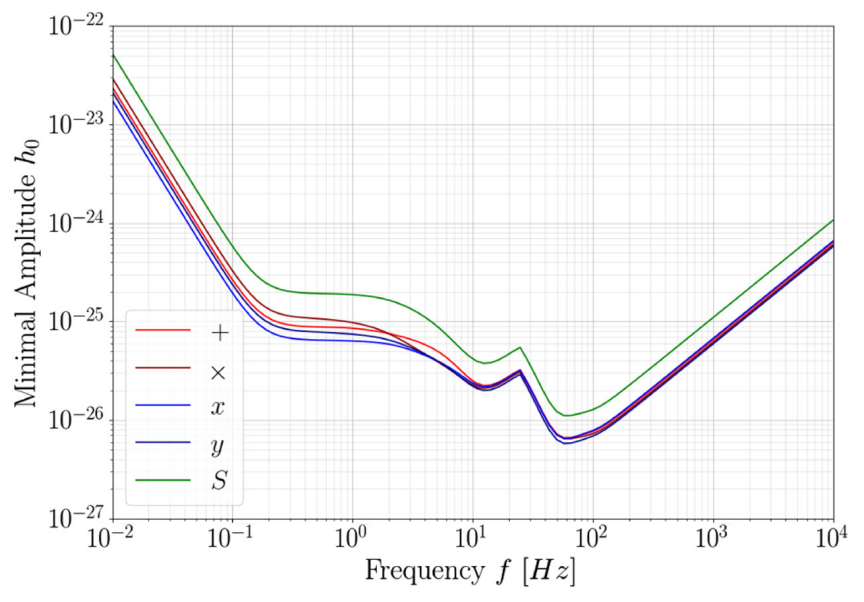

FIG. 9. Frequency-dependent sensitivity of the ET, LIGO, and DECIGO toward the polarizations $A \in\{+, \times, x, y, S\}$.

It turns out that the angular pattern functions of the breathing and the longitudinal modes are proportional to each other: $F_{I}^{l}=-\sqrt{2} F_{I}^{b}$. Therefore, it is impossible to distinguish these two with laser interferometry, and we thus focus on the distinction between the four tensor and vector

(a)

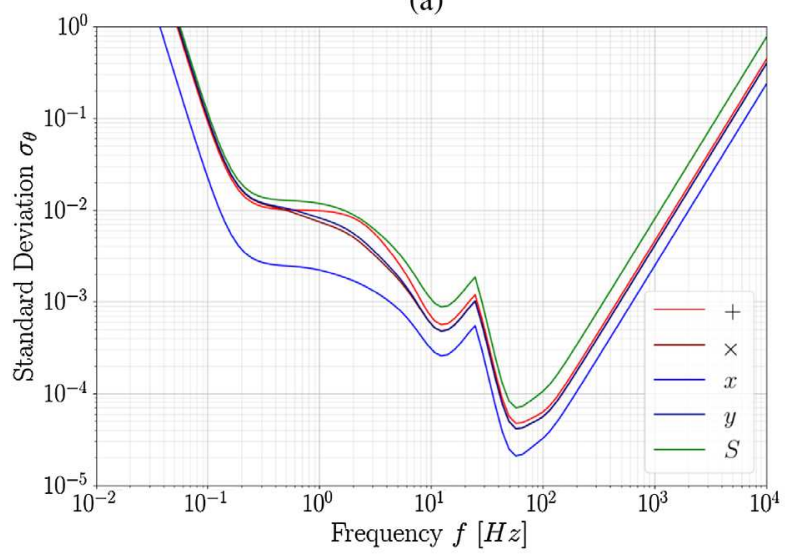

(c)

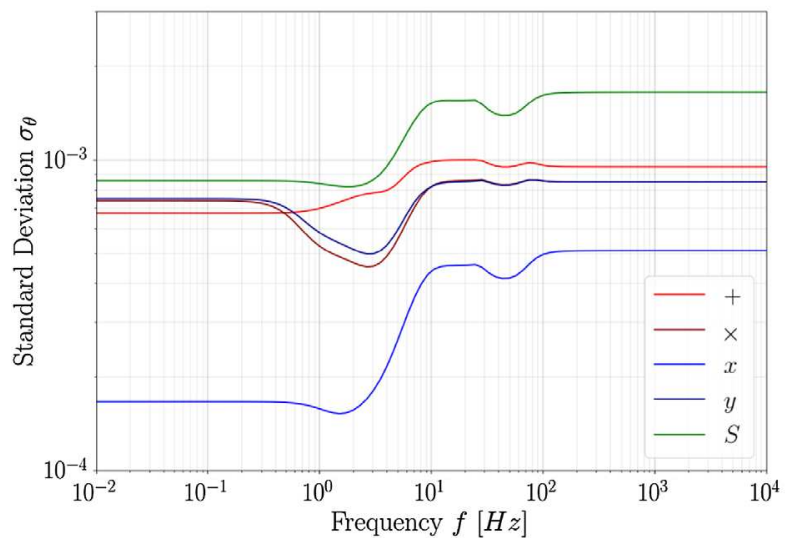

polarizations and the scalar mode. From now on, we use the polarization $A$ as

$$
A \in\{+, \times, x, y, S\} .
$$

In our calculations, we only use one cluster out of all the DECIGO clusters, namely, the one closest to Earth $\left(\phi=-20^{\circ}\right.$ from the Earth position, on its orbit around the Sun). We keep including the ET and the already existing LIGO detectors. We adopt the HEALPIX pixelization scheme to evenly distribute $n$ points on the sky (we used $n=48$ and $N_{\text {side }}=2$ to generate Fig. 9-13; more detailed explanations about the HEALPIX scheme can be found in Ref. [27]) and then average over the $h_{\min }$ values for each $f$ and obtain the frequency-dependant behavior of the average sensitivity of the ET, LIGO, and DECIGO in Fig. 9.

There are two aspects of the frequency-dependent standard deviation of $\theta$ and $\phi$. One is that if one measures a signal with a certain amplitude then we can measure the position of the source more precisely if it emits GW in frequencies in which the detector network is more sensitive. We plot this in Fig. 10. The other aspect is that the standard deviations vary with the frequency, relative to the sensitivity at that frequency. This means that if we consider, for

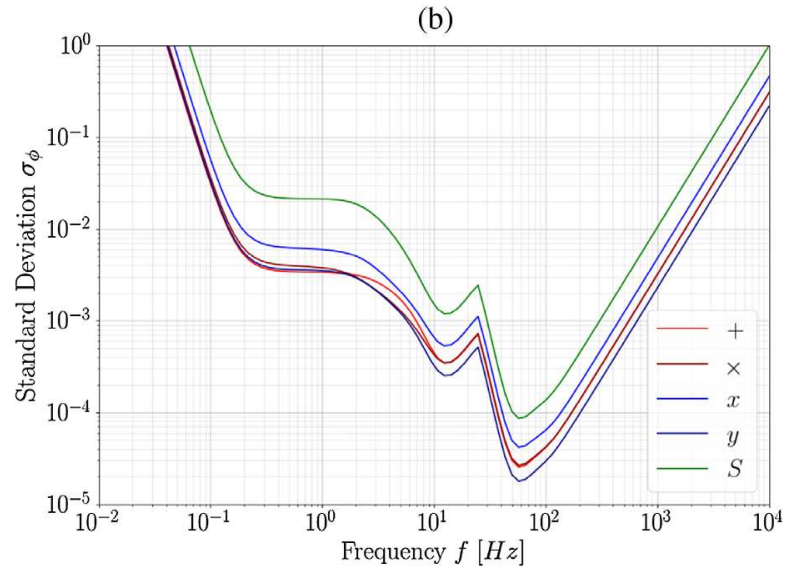

(d)

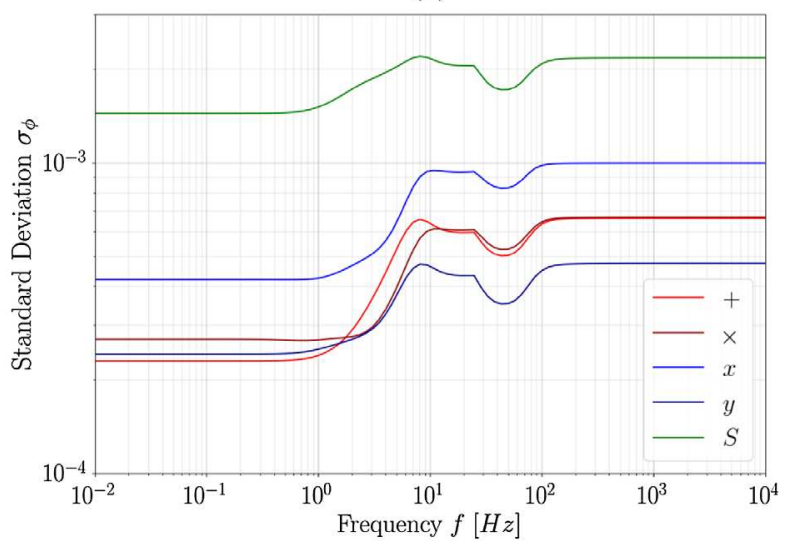

FIG. 10. Frequency-dependent standard deviation of $\theta$ and $\phi$ for (a),(b) a GW with amplitude $h=10^{-25}$ and (c),(d) twice the minimal amplitude of the scalar mode $h=2\left|h_{S}\right|_{\min }$ (see Fig. 9). 


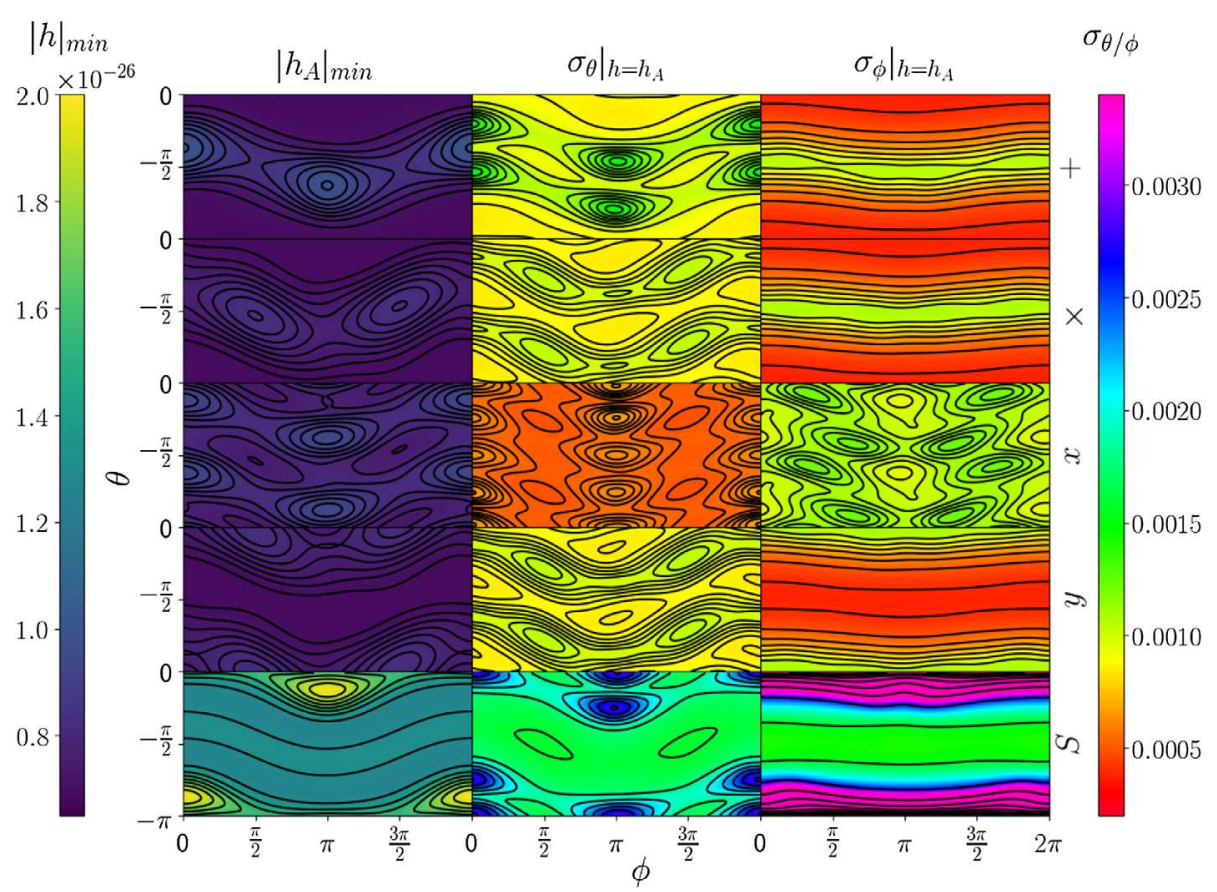

FIG. 11. Sensitivity of the ET, LIGO, and DECIGO at $100 \mathrm{~Hz}$ toward the polarizations $A \in\{+, \times, x, y, S\}$ in the left column. Standard deviation of the $\theta$ and $\phi$ angle for a GW with polarization $A$ and amplitude of $h_{A}=2.6 \times 10^{-26}$ in the middle and right columns, respectively.

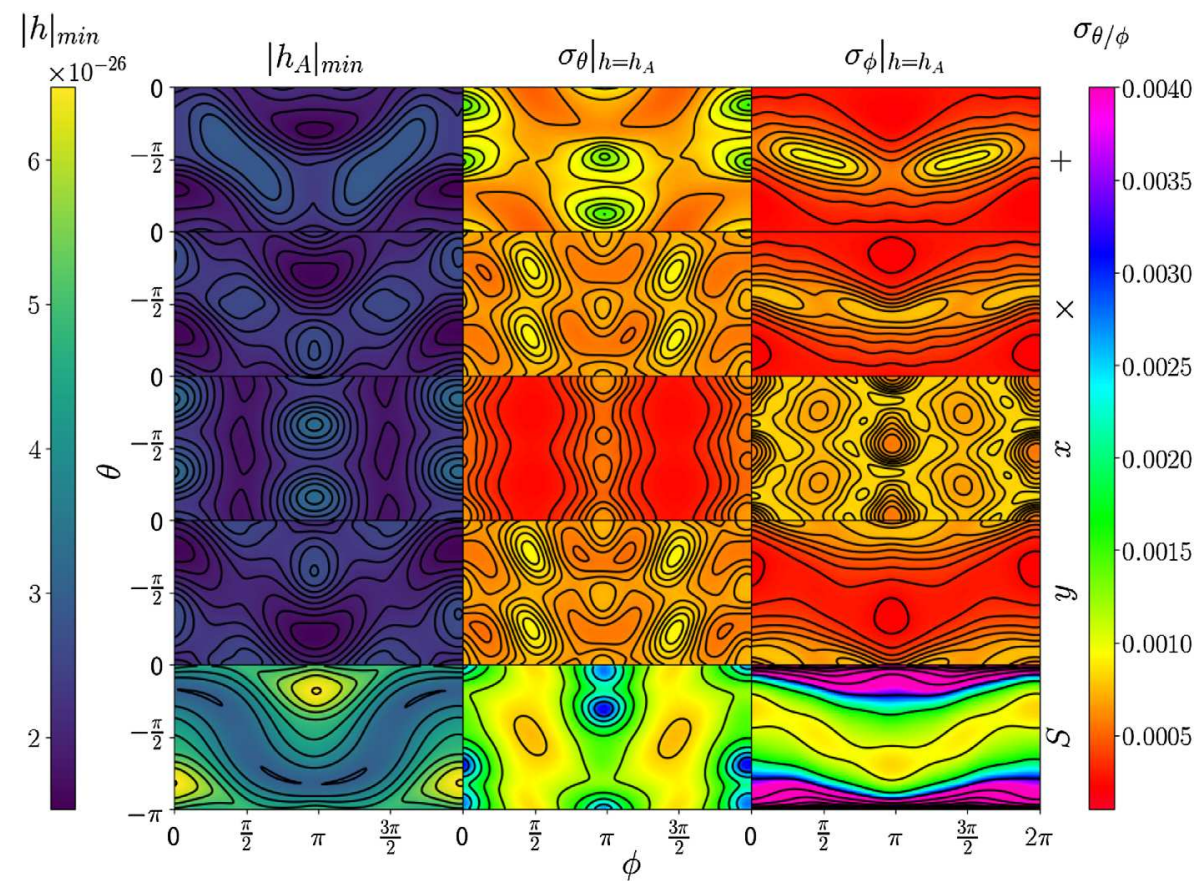

FIG. 12. Sensitivity of the ET, LIGO, and DECIGO at $10 \mathrm{~Hz}$ toward the polarizations $A \in\{+, \times, x, y, S\}$ in the left column. Standard deviation of the $\theta$ and $\phi$ angle for a GW with polarization $A$ and amplitude of $h_{A}=8.9 \times 10^{-26}$ in the middle and right columns, respectively.

instance, a wave that has twice the minimal amplitude for each frequency, we still get frequency dependence. In Fig. 10 (below), we take $h=2\left|h_{S}\right|_{\min }(f)$ since it is always higher than the other polarizations and we can therefore detect it, no matter which polarization we choose, and for any fixed frequency we have the same amplitude for all polarizations. This allows us to compare the polarizations with each other. 


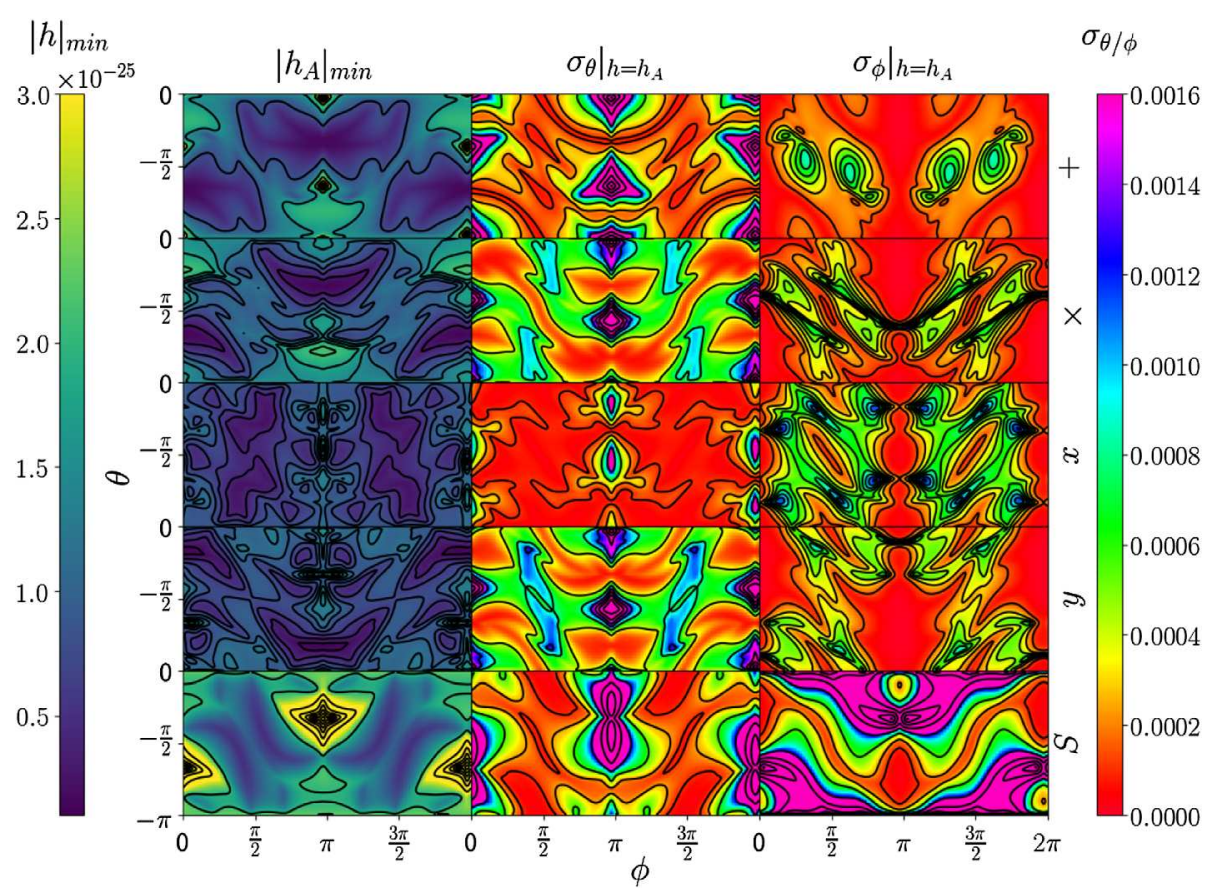

FIG. 13. Sensitivity of the ET, LIGO, and DECIGO at $1 \mathrm{~Hz}$ toward the polarizations $A \in\{+, \times, x, y, S\}$ in the left column. Standard deviation of the $\theta$ and $\phi$ angle for a GW with polarization $A$ and amplitude of $h_{A}=3.8 \times 10^{-25}$ in the middle and right columns, respectively.

In Fig. 11, we give the direction-dependent sensitivities using Eq. (76), along with the angular resolution for waves with these polarizations by taking the square root of Eqs. (77a) and (77b) at a frequency of $100 \mathrm{~Hz}$, where our set of detectors is most sensitive. We do a similar procedure at 10 and $1 \mathrm{~Hz}$, and the results are given in Figs. 12 and 13. Note that the purple zones correspond to true poles.

\section{CONCLUSION}

The Einstein Telescope alone cannot be used to distinguish between GW polarization modes, and small changes in its geometry lead to no almost no difference. However, by combining the ET with second-generation detectors such as LIGO, VIRGO, and KAGRA, one can detect a GW background with a strain amplitude down to $10^{-26}$ and distinguish its polarization modes around a frequency of $100 \mathrm{~Hz}$. One can enhance the sensitivity for lower frequencies by cross-correlating the network with the DECIGO detector, especially in its $\mathrm{C} 3$ configuration. In that case, the observation window is enhanced and allows measurement of strains below $10^{-23}$, down to $10^{-26}$, in a frequency band from 0.1 to $10 \mathrm{kHz}$.

It is possible to use an alternative method to distinguish the modes by using the time dependence of the signals. Using the right detector pairs, one can then clearly distinguish the scalar and vector modes by the blind spots. The effect is quite significant over a time period of one year for a ground-space network of detectors involving DECIGO, the ET, and LIGO. In the case of a scaled-down B-DECIGO detector, the time dependence is, however, very chaotic, due to the angular frequency around Earth, which is then an irrational fraction of Earth rotation, and the distance to Earth detectors, which varies significantly. The sensitivities of the different modes are too close together whenever the detectors are too close to Earth. This can be resolved, however, if one chooses an orbit on a higher altitude such that it circles the Earth once a day. In that case, the method becomes more complicated than with the original DECIGO but is still feasible.

We have thus showed that second- and third-generation detectors, combined with space detectors, can provide two different methods to test GR or constrain alternative theories by measuring the polarization of a GW background.

In a future project, one could investigate the possibilities of detecting inhomogeneities in the GW background, analogous to the ones in the cosmic microwave background. Up to now, we only calculated the minimal strain of a GW to be detected, but to find out how large the deviation from the mean would have to be to detect them, we would have to deal with the variance of the parameter estimation. It would be interesting to find out what angular resolution one could get with various detector combinations.

Gravitational waves should travel undisturbed since Planck time, which would make it possible to measure properties of the early quantum gravitational Universe directly. This could give us valuable hints on the search of a unifying theory. This feature of gravitational waves over electromagnetic waves also has its drawbacks. Because of the enormous density of the early Universe, many emissions of GW would be expected from different epochs after the big bang, and the difficulty 
would be to distinguish a signal of an earlier epoch from a later one. Overcoming that problem could, however, allow us to establish a complete gravitational map of the beginning of our Universe.

\section{ACKNOWLEDGMENTS}

L. P. was supported by the UZH research Grant No. FK17-097. P. J. thanks the Swiss National Foundation for support. We also thank the referee for the useful comments.

\section{APPENDIX A: SYMMETRY OF THE ET}

\section{ET perturbations}

We have seen in Sec. III that one cannot distinguish between the three polarization modes with the ET alone, although we have three signals, because of the symmetric arrangement of the three interferometers composing the ET. One has to thus break the symmetry in order to make the three rows in the detector correlation matrix independent. We are going to consider two ways of doing that perturbatively and use the framework of the previous section to determine their impact on the sensitivity, which will allow us to compare both methods.

\section{a. Irregular triangle}

We make one opening angle smaller by a small angle $\epsilon_{\phi}$ and make another angle bigger by the same amount, which leaves the third angle unchanged. We now have a completely irregular triangle with three different angles, and therefore the ORFs of all three detector pairs become different, and the detector correlation matrix becomes invertible.
Changing the angles will also change the arm lengths and therefore the distance between a detector pair. We use the sine law to determine the impact of a change in the angles on the change in the distance $\delta$. To estimate the order of magnitude of the effect of the perturbation on the detector correlation matrix, we calculate the change in the ORF of the detector pair $(1,2)$, when we shrink the angle $\phi_{3}$ and enlarge $\phi_{1}$ by $\epsilon_{\phi}$, which leaves $\phi_{2}$ unchanged but shortens $d_{12}$,

$$
\frac{d-\delta}{\sin \left(\phi_{3}\right)}=\frac{d}{\sin \left(\phi_{1}\right)}, \quad \phi_{3}=\phi-\epsilon_{\phi}, \quad \phi_{1}=\phi+\epsilon_{\phi},
$$

for $\phi=\pi / 3$. To first order, we get

$$
\delta \approx \sqrt{3} d \epsilon_{\phi} .
$$

With that expression, we can relate the effects of a change in the distance to the change in the angles:

$$
\begin{gathered}
\rho_{i}^{M} \mapsto\left(\rho_{i}^{M}\right)^{(0)}-\sqrt{3} \alpha\left(\rho_{i}^{M}\right)^{\prime} \epsilon_{\phi}, \\
\cos \beta \mapsto \cos \beta^{(0)}+\sqrt{3} \frac{d^{2}}{R_{E}^{2}} \epsilon_{\phi} .
\end{gathered}
$$

The only coordinate angle that changes is $\sigma_{1+}$. If we now insert in Eq. (38) the values of all angles,

$$
\begin{gathered}
\sigma_{1-}=0, \quad \sigma_{1+} \mapsto \sigma_{1+}^{(0)}+\epsilon_{\phi}, \quad \sigma_{1+}^{(0)}=\frac{\pi}{3}, \\
\sigma_{2-}=\frac{2 \pi}{3}, \quad \sigma_{2+}=\pi,
\end{gathered}
$$

we get the new ORF:

$$
\begin{aligned}
\gamma_{12}^{M}= & \frac{1}{16}\left\{-3 \rho_{1}^{M} \sin ^{2} \sigma_{1+}+\frac{\sqrt{3}}{2}\left[2 \rho_{1}^{M} \cos \beta+\rho_{2}^{M} \frac{1+\cos \beta}{2}\right] \sin \left(2 \sigma_{1+}\right)\right. \\
& \left.+\frac{3}{4}\left[4 \rho_{1}^{M} \cos ^{2} \beta+2 \rho_{2}^{M}(1+\cos \beta) \cos \beta+\rho_{3}^{M}(1+\cos \beta)^{2}\right]\left(\cos ^{2} \sigma_{1+}-1\right)\right\} \\
\mapsto & \frac{1}{16}\left\{-3\left(\left(\rho_{1}^{M}\right)^{(0)}-\sqrt{3} \alpha\left(\rho_{1}^{M}\right)^{\prime} \epsilon_{\phi}\right)\left(\sin ^{2} \sigma_{1+}^{(0)}+2 \sin \sigma_{1+}^{(0)} \cos \sigma_{1+}^{(0)} \epsilon_{\phi}\right)\right. \\
& +\frac{\sqrt{3}}{2}\left[2\left(\left(\rho_{1}^{M}\right)^{(0)}-\sqrt{3} \alpha\left(\rho_{1}^{M}\right)^{\prime} \epsilon_{\phi}\right)\left(\cos \beta^{(0)}+\sqrt{3} \frac{d^{2}}{R_{E}^{2}} \epsilon_{\phi}\right)\right. \\
& \left.+\frac{1}{2}\left(\left(\rho_{2}^{M}\right)^{(0)}-\sqrt{3} \alpha\left(\rho_{2}^{M}\right)^{\prime} \epsilon_{\phi}\right)\left(1+\cos \beta^{(0)}+\sqrt{3} \frac{d^{2}}{R_{E}^{2}} \epsilon_{\phi}\right)\right]\left(\sin \left(2 \sigma_{1+}^{(0)}\right)+2 \cos \left(2 \sigma_{1+}^{(0)}\right) \epsilon_{\phi}\right) \\
& +\frac{3}{4}\left[4\left(\left(\rho_{1}^{M}\right)^{(0)}-\sqrt{3} \alpha\left(\rho_{1}^{M}\right)^{\prime} \epsilon_{\phi}\right)\left(\cos \beta^{(0)}+\sqrt{3} \frac{d^{2}}{R_{E}^{2}} \epsilon_{\phi}\right)^{2}\right. \\
& +2\left(\left(\rho_{2}^{M}\right)^{(0)}-\sqrt{3} \alpha\left(\rho_{2}^{M}\right)^{\prime} \epsilon_{\phi}\right)\left(1+\cos \beta^{(0)}+\sqrt{3} \frac{d^{2}}{R_{E}^{2}} \epsilon_{\phi}\right)\left(\cos \beta^{(0)}+\sqrt{3} \frac{d^{2}}{R_{E}^{2}} \epsilon_{\phi}\right) \\
& \left.\left.+\left(\left(\rho_{3}^{M}\right)^{(0)}-\sqrt{3} \alpha\left(\rho_{3}^{M}\right)^{\prime} \epsilon_{\phi}\right)\left(1+\cos \beta^{(0)}+\sqrt{3} \frac{d^{2}}{R_{E}^{2}} \epsilon_{\phi}\right)^{2}\right]\left(\cos \sigma_{1+}^{(0)}-2 \cos \sigma_{1+}^{(0)} \sin \sigma_{1+}^{(0)} \epsilon_{\phi}-1\right)\right\}
\end{aligned}
$$


We can simplify this expression, by plugging in the values for $\sigma_{1+}^{(0)}$ and approximating $\cos \beta^{(0)}$, using the fact that $\frac{d^{2}}{R_{E}^{2}}=2.5 \times 10^{-6} \ll 1:$

$$
\cos \beta^{(0)}=1-\frac{d^{2}}{2 R_{E}^{2}} \approx 1
$$

To first order in $\epsilon_{\phi}$, we get

$$
\begin{aligned}
\epsilon_{M} \approx & \frac{1}{16}\left\{-\frac{3 \sqrt{3}}{2}\left[\left(\rho_{1}^{M}\right)^{(0)}-\frac{3}{2} \alpha\left(\rho_{1}^{M}\right)^{\prime}\right]-\frac{\sqrt{3}}{2}\left[2\left(\rho_{1}^{M}\right)^{(0)}+3 \alpha\left(\rho_{1}^{M}\right)^{\prime}+\left(\rho_{2}^{M}\right)^{(0)}+\frac{3}{2} \alpha\left(\rho_{2}^{M}\right)^{\prime}\right]\right. \\
& \left.-\frac{3 \sqrt{3}}{4}\left[2\left(\left(\rho_{1}^{M}\right)^{(0)}+\left(\rho_{2}^{M}\right)^{(0)}+\left(\rho_{3}^{M}\right)^{(0)}\right)-3 \alpha\left(\left(\rho_{1}^{M}\right)^{\prime}+\left(\rho_{2}^{M}\right)^{\prime}+\left(\rho_{3}^{M}\right)^{\prime}\right)\right]\right\} \epsilon_{\phi} \\
\approx & -\frac{\sqrt{3}}{32}\left\{8\left(\rho_{1}^{M}\right)^{(0)}+4\left(\rho_{2}^{M}\right)^{(0)}+3\left(\rho_{3}^{M}\right)^{(0)}-3 \alpha\left(2\left(\rho_{1}^{M}\right)^{\prime}+\left(\rho_{2}^{M}\right)^{\prime}+\frac{3}{2}\left(\rho_{3}^{M}\right)^{\prime}\right)\right\} \epsilon_{\phi} .
\end{aligned}
$$

In Fig. 14, we plot the response factor, which multiplies to $\epsilon_{\phi}$ to get the change in the ORF $\epsilon_{M}$.

The response factor stays almost constant at a value of -0.87 , since $\alpha \ll 1$ until we get close to the critical frequency $f_{\text {crit }}=3 \times 10^{4} \mathrm{~Hz}$, defined over $\frac{f_{\text {crit }} d}{c}:=1$.

\section{b. Tilted detector planes}

Now, we leave the angles and the arm lengths of the three Michelson interferometers invariant but tilt the plane in which one of the three detectors lies. We tilt the plane of detector 1 , such that $\hat{u}_{1}$ gets tilted in negative $z$ direction. The other detector arms stay unchanged $\left(\hat{u}_{J}=\hat{u}_{J}^{(0)}\right.$ for $J \neq 1$ and $\hat{v}_{J}=\hat{v}_{J}^{(0)}$ for all $\left.J\right)$, and we can write the perturbation as

$$
\hat{u}_{1} \mapsto \hat{u}_{1}^{(0)}+\delta \hat{u}_{1}, \quad \delta \hat{u}_{1}=\left(\begin{array}{c}
0 \\
0 \\
-\delta u
\end{array}\right) .
$$

The angle $\alpha$ by which $\hat{u}_{1}$ is rotated can be approximated by

$$
\alpha \approx \sin \alpha \approx \delta u
$$

We calculate the contractions of the perturbed detector tensors, analogous to Eqs. (35)-(37) to first order in $\delta u$ :

$$
\begin{aligned}
D_{1}^{i j} D_{i j}^{2} & =\frac{1}{4}\left[\left(\hat{u}_{1}^{(0)} \cdot \hat{u}_{2}+\delta \hat{u}_{1} \cdot \hat{u}_{2}\right)^{2}-\left(\hat{v}_{1} \cdot \hat{u}_{2}\right)^{2}-\left(\hat{u}_{1}^{(0)} \cdot \hat{v}_{2}+\delta \hat{u}_{1} \cdot \hat{v}_{2}\right)^{2}+\left(\hat{v}_{1} \cdot \hat{v}_{2}\right)^{2}\right] \\
& \approx \frac{1}{4}\left(D_{1}^{i j} D_{i j}^{2}\right)^{(0)}+\frac{1}{2}\left[\left(\hat{u}_{1}^{(0)} \cdot \hat{u}_{2}\right)\left(\delta \hat{u}_{1} \cdot \hat{u}_{2}\right)-\left(\hat{u}_{1}^{(0)} \cdot \hat{v}_{2}\right)\left(\delta \hat{u}_{1} \cdot \hat{v}_{2}\right)\right] .
\end{aligned}
$$

Using the angles for the ET as in Eq. (A6), we get

$$
\begin{aligned}
\delta\left(D_{1}^{i j} D_{i j}^{2}\right)= & \frac{1}{2}\left[\left(\cos \beta \cos \sigma_{1-} \cos \sigma_{2-}+\sin \sigma_{1-} \sin \sigma_{2-}\right) \delta u \sin \beta \cos \sigma_{2-}\right. \\
& \left.-\left(\cos \beta \cos \sigma_{1-} \cos \sigma_{2+}+\sin \sigma_{1-} \sin \sigma_{2+}\right) \delta u \sin \beta \cos \sigma_{2+}\right] \\
= & \frac{1}{2} \cos \beta \sin \beta\left(\cos ^{2} \sigma_{2-}-1\right) \delta u=-\frac{3}{8} \cos \beta \sin \beta \delta u
\end{aligned}
$$




$$
\begin{aligned}
D_{1, k}^{i} D_{2}^{k j} \hat{d}_{i} \hat{d}_{j} & =\frac{1}{4}\left(\left(\hat{u}_{1}^{(0)} \cdot \hat{d}+\delta \hat{u}_{1} \cdot \hat{d}\right)\left(\hat{u}_{1}^{(0)}+\delta \hat{u}_{1}\right)-\left(\hat{v}_{1} \cdot \hat{d}\right) \hat{v}_{1}\right) \cdot\left(\left(\hat{u}_{2} \cdot \hat{d}\right) \hat{u}_{2}-\left(\hat{v}_{2} \cdot \hat{d}\right) \hat{v}_{2}\right) \\
& \approx\left(D_{1, k}^{i} D_{2}^{k j} \hat{d}_{i} \hat{d}_{j}\right)^{(0)}+\frac{1}{4}\left(\left(\delta \hat{u}_{1} \cdot \hat{d}\right) \hat{u}_{1}^{(0)}+\left(\hat{u}_{1}^{(0)} \cdot \hat{d}\right) \delta \hat{u}_{1}\right) \cdot\left(\left(\hat{u}_{2} \cdot \hat{d}\right) \hat{u}_{2}-\left(\hat{v}_{2} \cdot \hat{d}\right) \cdot \hat{v}_{2}\right), \\
\delta\left(D_{1, k}^{i} D_{2}^{k j} \hat{d}_{i} \hat{d}_{j}\right) & =\frac{1}{8(1-\cos \beta)}\left(\delta u(1-\cos \beta) \hat{u}_{1}^{(0)}+\cos \sigma_{1-} \sin \beta \delta \hat{u}_{1}\right) \cdot\left(\sin \beta \cos \sigma_{2-} \hat{u}_{2}-\sin \beta \cos \sigma_{2+} \hat{v}_{2}\right) \\
& =\frac{\sin \beta}{8}(\cos \sigma_{2-}-\underbrace{\cos \beta \cos \sigma_{2-}}_{\hat{u}_{1}^{(0)} \cdot \hat{u}_{2}}-\underbrace{\cos \beta}_{\hat{u}_{1}^{(0)} \cdot \hat{v}_{2}}) \delta u+\frac{1+\cos \beta}{8}(\cos \sigma_{2-} \delta u \sin \beta \cos \sigma_{2-}-\underbrace{\delta u \sin \beta}_{\delta \hat{u}_{1} \cdot \hat{v}_{2}}) \\
& =\frac{\sin \beta}{8}\left[\cos \beta\left(\cos ^{2} \sigma_{2-}-1\right)+(1+\cos \beta)\left(\cos ^{2} \sigma_{2-}-1\right)\right] \delta u \\
& =-\frac{\sin \beta}{8}(1+2 \cos \beta) \sin ^{2} \sigma_{2-} \delta u=-\frac{3}{4} \frac{\sin \beta}{8}(1+2 \cos \beta) \delta u, \\
D_{1}^{i j} D_{2}^{k l} \hat{d}_{i} \hat{d}_{j} \hat{d}_{k} \hat{d}_{l} & =\frac{1}{4}\left(\left(\hat{u}_{1}^{(0)} \cdot \hat{d}+\delta \hat{u}_{1} \cdot \hat{d}\right)^{2}-\left(\hat{v}_{1} \cdot \hat{d}\right)^{2}\right)\left(\left(\hat{u}_{2} \cdot \hat{d}\right)^{2}-\left(\hat{v}_{2} \cdot \hat{d}\right)^{2}\right) \\
& =\left(D_{1}^{i j} D_{2}^{k l} \hat{d}_{i} \hat{d}_{j} \hat{d}_{k} \hat{d}_{l}\right)^{(0)}+\frac{1}{2}\left(\left(\hat{u}_{1}^{(0)} \cdot \hat{d}\right)\left(\delta \hat{u}_{1} \cdot \hat{d}\right)\right)\left(\left(\hat{u}_{2} \cdot \hat{d}\right)^{2}-\left(\hat{v}_{2} \cdot \hat{d}\right)^{2}\right), \\
\delta\left(D_{1}^{i j} D_{2}^{k l} \hat{d}_{i} \hat{d}_{j} \hat{d}_{k} \hat{d}_{l}\right) & =\frac{1}{8(1-\cos \beta)^{2}} \cos \sigma_{1-} \sin \beta \delta u(1-\cos \beta)\left(\sin ^{2} \beta \cos ^{2} \sigma_{2-}-\sin ^{2} \beta \cos ^{2} \sigma_{2+}\right) \\
& =\frac{1+\cos \beta}{8} \sin \beta\left(\cos { }^{2} \sigma_{2-}-1\right) \delta u=-\frac{3}{4} \frac{1+\cos \beta}{8} \sin \beta \delta u ._{(\mathrm{A} 14)}
\end{aligned}
$$

Finally, we can patch all terms together in order to calculate the perturbation $\epsilon_{M}$ :

$$
\begin{aligned}
\epsilon_{M} & =\rho_{1}^{M} \delta\left(D_{1}^{i j} D_{i j}^{2}\right)+\rho_{2}^{M} \delta\left(D_{1, k}^{i} D_{2}^{k j} \hat{d}_{i} \hat{d}_{j}\right)+\rho_{3}^{M} \delta\left(D_{1}^{i j} D_{2}^{k l} \hat{d}_{i} \hat{d}_{j} \hat{d}_{k} \hat{d}_{l}\right) \\
& =-\frac{3}{8} \sin \beta\left\{\rho_{1}^{M} \cos \beta+\frac{1}{4} \rho_{2}^{M}(1+2 \cos \beta)+\frac{1}{4} \rho_{3}^{M}(1+\cos \beta)\right\} \delta u .
\end{aligned}
$$

Again, we find that $\epsilon_{M}$ is almost independent of $f$, but the effect is 3 orders of magnitude smaller if we tilt one plane, instead of deforming the equilateral triangle,

$$
\epsilon_{M}=1.2 \times 10^{-3} \delta u
$$

The response factor for the tilted plane, shown in Fig. 15, stays at about -0.001 for frequencies far below $f_{\text {crit }}$ and oscillates ever closer around zero for increasing frequencies above $10^{5} \mathrm{~Hz}$. Since the ET is designed to measure a

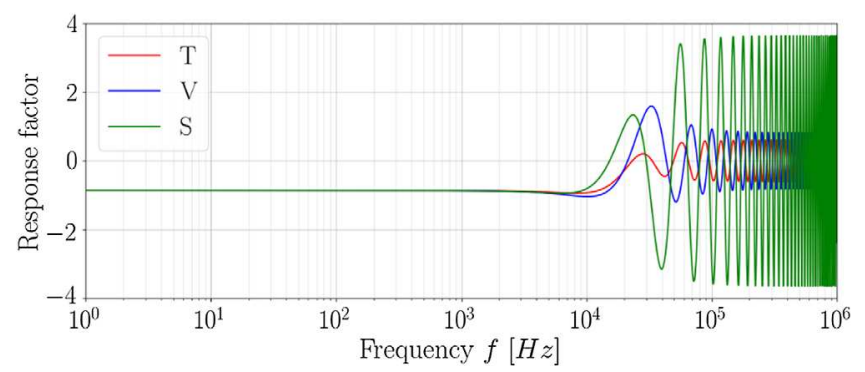

FIG. 14. The factor with which the ORF responds to a small change in the detector angles. frequency range from 1.5 to $10 \mathrm{kHz}$, the oscillations are not relevant. We find that the response to the same small change in the tilt angle is 3 orders of magnitude smaller than that of the change in the opening angle.

The effect of a perturbation is at best as small as the angle by which we change the ET's geometry, in the case of the irregular triangle. As we will argue in the next section, the problem is resolved if one adds additional detectors, e.g., LIGO, which exists already anyway, and changing the geometry of the ET is therefore not worth the effort.

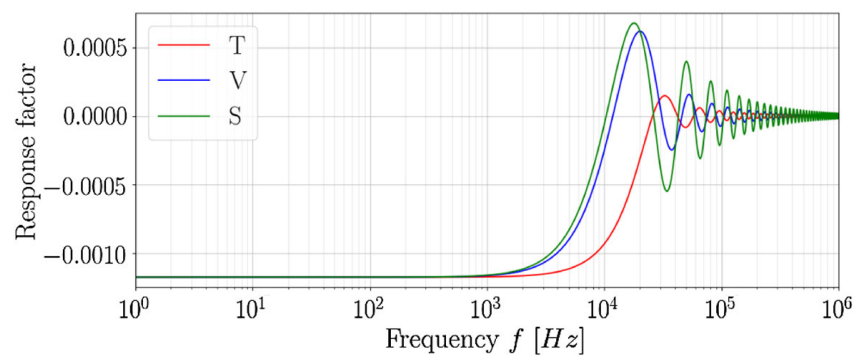

FIG. 15. Factor with which the ORF responds to a small tilt of one of the detector planes. 


\section{APPENDIX B: B-DECIGO}

The scaled-down detector B-DECIGO [19] orbits around the Earth on an altitude of $2000 \mathrm{~km}$, which is on the same order of magnitude as the radius of the Earth $(6371 \mathrm{~km})$. If we replace DECIGO by B-DECIGO, we can see in Fig. 16 that the sensitivity gets worse below $10 \mathrm{~Hz}$ for all polarizations, as compared to DECIGO.

B-DECIGO circles the Earth in a Sun-synchronous dusk-dawn orbit with an angular frequency of about $8.2 \times 10^{-4} \mathrm{~s}^{-1}$, while Earth rotation corresponds to $7.3 \times 10^{-5} \mathrm{~s}^{-1}$. This leads to rapidly varying distances and directions of the detector arms, and the irrational ratio between the two angular velocities leads to a chaotic behaviour, which makes the use of the time-dependent sensitivity very complicated. Additionally, one can observe that the sensitivities for the different modes get closer together as one moves a space detector closer to Earth.

If one would instead let B-DECIGO take the same type of orbit but on a higher altitude $(35,867 \mathrm{~km})$, such that it would circle Earth in one day, one would get almost the same signal every day over a period of about a week, because the change would now be on the timescale of a year. The detectors would also be far enough from Earth to get relevantly different sensitivity curves for the different modes. The procedure would be more complicated than in the case of DECIGO, but one could still use certain blind spots or other characteristics that only one mode shows. A large disadvantage to DECIGO would also be that one would have to spot those characteristics in a model in advance, since the sensitivities are not periodic.

We compare the time-dependent sensitivities of both versions (original B-DECIGO and higher altitude) for time span of one day in Fig. 17.

In Fig. 18, we plot the frequency-dependent sensitivity of B-DECIGO together with the ET and LIGO in the case of point sources. The behavior is very similar to that with DECIGO, except that the plateau around $1 \mathrm{~Hz}$ is missing.

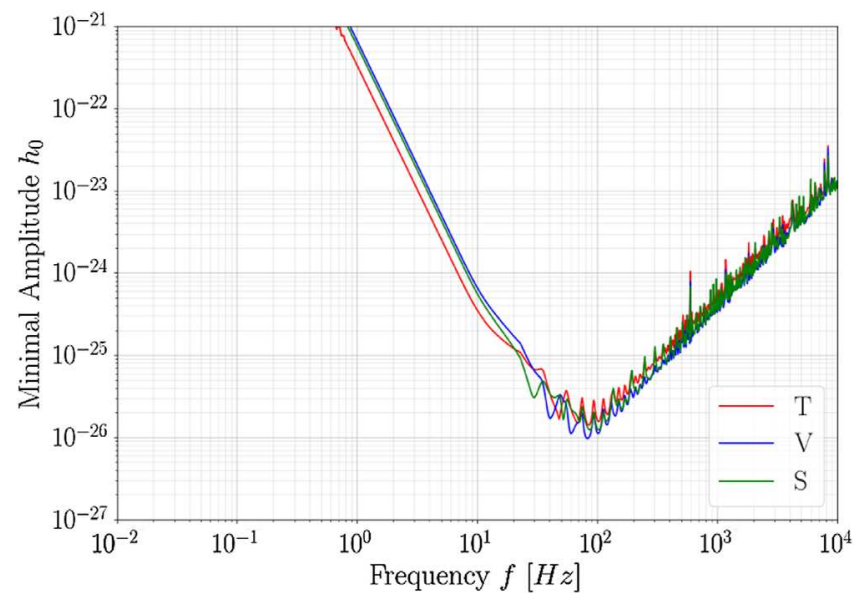

FIG. 16. Combined sensitivity of B-DECIGO, the ET, and both advanced LIGO detectors.
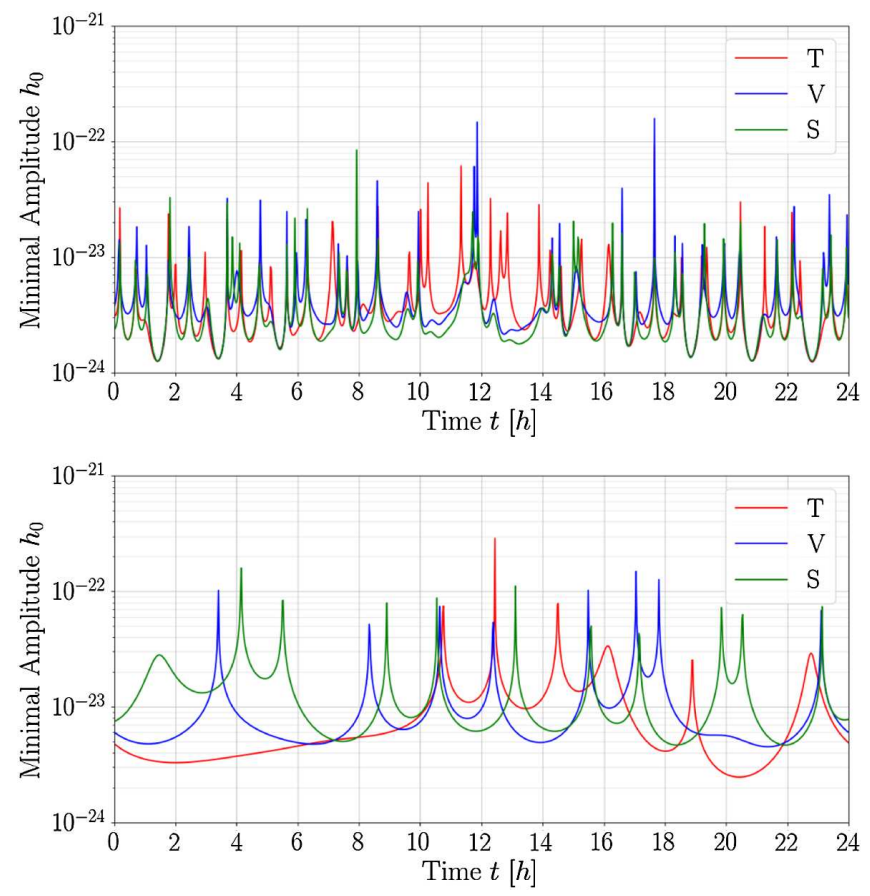

FIG. 17. Time dependence of the sensitivity for B-DECIGO for one day (above) and for a higher altitude of $35867 \mathrm{~km}$ (below) for a frequency of $100 \mathrm{~Hz}$.

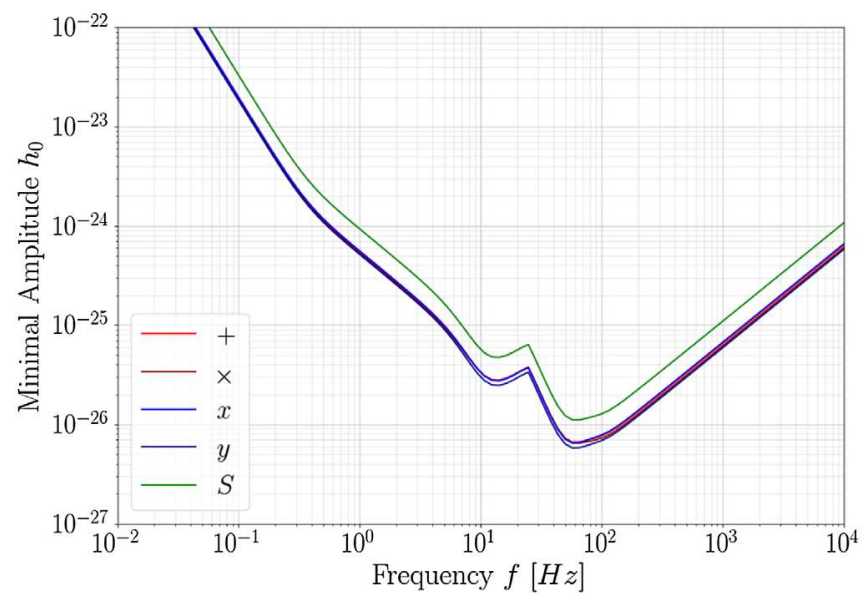

FIG. 18. Frequency-dependent sensitivity of the ET, LIGO, and B-DECIGO.

Since B-DECIGO is not as sensitive as DECIGO, it can only increase the sensitivity there a bit.

\section{APPENDIX C: DELTA DISTRIBUTION APPROXIMATION}

In this Appendix, we give a detailed derivation of the signal-to-noise ratio for a merger by focusing on the approximations of the Dirac delta distribution and the Fourier transforms. We first use a scalar signal, measured by two detectors, to simplify the calculation and then 
generalize to a wave with arbitrary polarizations measured by multiple detectors.

In the future, all GW detectors together could be sensitive enough to measure the inspiral of a binary black hole or neutron star merger, months before the merger event happens. In this case, detectors with different distances from the source would have different observation times. This would help in measuring the position of the source in the sky,

$$
c \Delta T=\hat{\Omega} \cdot \Delta \vec{x}_{I J}, \quad \Delta T=T_{I}-T_{J}, \quad \Delta \vec{x}_{I J}=\vec{x}_{I}-\vec{x}_{J},
$$

where $T_{I}$ and $T_{J}$ are the observation times of the detectors $I$ and $J, \vec{x}_{I}$ and $\vec{x}_{J}$ are their position vectors, and $\hat{\Omega}$ is the direction of travel of the GW.
We define the cross-correlated and filtered strain amplitude of the detector pair $(I, J)$ by

$$
Y:=\int_{-T_{I} / 2}^{T_{I} / 2} \int_{-T_{J} / 2}^{T_{J} / 2} s_{I}(t) s_{J}\left(t^{\prime}\right) Q\left(t-t^{\prime}\right) d t^{\prime} d t
$$

where $Q$ is the filter function and $s_{I}$ and $s_{J}$ are the strains measured by the detectors $I, J$, which are the sum of the signal $h_{I}$ and the noise $n_{I}$ in detector $I$ :

$$
s_{I}(t)=h_{I}(t)+n_{I}(t) .
$$

By taking the ensemble average, we get rid of the noise terms,

$$
\begin{aligned}
& \mu:=\mathbb{E}[Y] \\
& =\int_{-T_{I} / 2}^{T_{I} / 2} \int_{-T_{J} / 2}^{T_{J} / 2}\left\{\mathbb{E}\left[h_{I}(t) h_{J}\left(t^{\prime}\right)\right]+\mathbb{E}\left[h_{I}(t) n_{J}\left(t^{\prime}\right)\right]+\mathbb{E}\left[n_{I}(t) h_{J}\left(t^{\prime}\right)\right]+\mathbb{E}\left[n_{I}(t) n_{J}\left(t^{\prime}\right)\right]\right\} Q\left(t-t^{\prime}\right) d t^{\prime} d t \\
& =\int_{-T_{I} / 2}^{T_{I} / 2} \int_{-T_{J} / 2}^{T_{J} / 2} \int \tilde{h}_{I}^{*}(f) e^{2 \pi i f\left(t-\frac{\hat{\hat{\hat{S}}} \cdot \vec{x}_{I}}{c}\right)} d f \int \tilde{h}_{J}\left(f^{\prime}\right) e^{-2 \pi i f^{\prime}\left(t^{\prime}-\frac{\hat{\hat{s}} \cdot \vec{x}_{J}}{c}\right)} d f^{\prime} Q\left(t-t^{\prime}\right) d t^{\prime} d t
\end{aligned}
$$

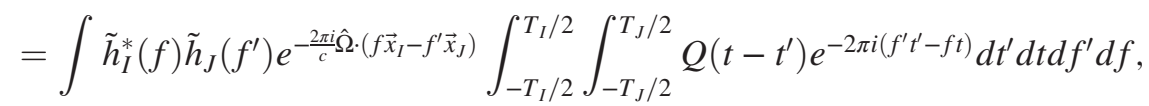

where we replaced the signal by its Fourier transform: $h_{I}(t)=\int \tilde{h}_{I}(f) e^{-2 \pi i f\left(t-\frac{\hat{\Omega} \cdot \vec{x}}{c}\right)} d f$.

We apply the substitution to the integral over $t^{\prime}, \tau=t-t^{\prime}, d \tau=d t$,

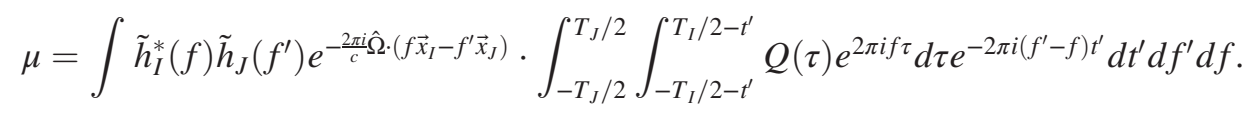

Then, we approximate the integral over $\tau$ with the Fourier transform of the filter function $Q$ :

$$
\int_{-T_{I} / 2-t^{\prime}}^{T_{I} / 2-t^{\prime}} Q(\tau) e^{2 \pi i f \tau} d \tau \approx \tilde{Q}(f)
$$

If we shift a wave packet in time, it is still composed of the same frequencies. Therefore, we can ignore the time shift in the integration volume by $-t$.

We pull this out of the $t$ integral and get

$$
\int_{-T_{J} / 2}^{T_{J} / 2} e^{-2 \pi i\left(f^{\prime}-f\right) t^{\prime}} d t^{\prime}=-\frac{1}{\pi \Delta f} \frac{1}{2 i}\left(e^{-\pi i \Delta f T_{J}}-e^{\pi i \Delta f T_{J}}\right)=\frac{\sin \left(\pi \Delta f T_{J}\right)}{\pi \Delta f}=: \delta_{T_{J}}\left(f^{\prime}-f\right) .
$$

If $\Delta f=f^{\prime}-f$ approaches zero, we get $\lim _{\Delta f \rightarrow 0} \delta_{T_{J}}(\Delta f)=\lim _{\Delta f \rightarrow 0} \frac{1}{\pi \Delta f}\left(0+\pi T_{J} \Delta f+\mathcal{O}\left(\Delta f^{2}\right)\right)=T_{J}$, and for big $\Delta f$, $\delta_{T_{J}}$ gets small:

$$
\left|\frac{\sin \left(\pi \Delta f T_{J}\right)}{\pi \Delta f}\right| \leq \frac{1}{\pi \Delta f} \stackrel{\Delta f \rightarrow \infty}{\longrightarrow} 0
$$


By approximating $\delta_{T_{J}}\left(f^{\prime}-f\right) \approx \delta\left(f^{\prime}-f\right)$ with the Dirac delta distribution, we can evaluate the integral over $f^{\prime}$ :

$$
\mu \approx \int \tilde{h}_{I}^{*}(f) \tilde{h}_{J}\left(f^{\prime}\right) e^{-\frac{2 \pi i}{c} \hat{\hat{Q}} \cdot\left(f \vec{x}_{I}-f^{\prime} \vec{x}_{J}\right)} \tilde{Q}\left(f^{\prime}\right) \delta\left(f^{\prime}-f\right) d f^{\prime} d f=\int \tilde{h}_{I}^{*}(f) \tilde{h}_{J}(f) \tilde{Q}(f) e^{-2 \pi i f \frac{\hat{\hat{\Omega}} \cdot \vec{\Delta}_{I J}}{c}} d f
$$

We now have an expression for the signal. To calculate the signal-to-noise ratio, we need to deal with noise, which is the square root of the variance in the absence of a signal:

$$
\begin{aligned}
\sigma^{2} & :=\left.\mathbb{V}[Y]\right|_{h=0}=\mathbb{E}\left[Y^{2}\right]-\left.\mathbb{E}[Y]^{2}\right|_{h=0}=\left.\mathbb{E}\left[Y^{2}\right]\right|_{h=0} \\
& =\left.\int_{-T_{I} / 2}^{T_{I} / 2} \int_{-T_{I} / 2}^{T_{I} / 2} \int_{-T_{J} / 2}^{T_{J} / 2} \int_{-T_{J} / 2}^{T_{J} / 2} \mathbb{E}\left[s_{I}(t) s_{I}\left(t^{\prime}\right) s_{J}(\tau) s_{J}\left(\tau^{\prime}\right)\right] Q(t-\tau) Q\left(t^{\prime}-\tau^{\prime}\right) d \tau^{\prime} d \tau d t^{\prime} d t\right|_{h=0} \\
& =\int_{-T_{I} / 2}^{T_{I} / 2} \int_{-T_{I} / 2}^{T_{I} / 2} \int_{-T_{J} / 2}^{T_{J} / 2} \int_{-T_{J} / 2}^{T_{J} / 2} \mathbb{E}\left[n_{I}(t) n_{I}\left(t^{\prime}\right)\right] \mathbb{E}\left[n_{J}(\tau) n_{J}\left(\tau^{\prime}\right)\right] Q(t-\tau) Q\left(t^{\prime}-\tau^{\prime}\right) d \tau^{\prime} d \tau d t^{\prime} d t
\end{aligned}
$$

Since the noises of the two detectors are independent of each other, we can take their expectation separately. We then insert the FT of the noise, in the time interval in which the measurement is taken,

$$
n_{I}(t)=\int \tilde{n}_{I}(f) e^{-2 \pi i f t} d f
$$

and then swap the time and frequency integrals and approximate the FT of the filter function and the delta distribution as before:

$$
\begin{aligned}
\sigma^{2}= & \int \mathbb{E}\left[\tilde{n}_{I}^{*}(f) \tilde{n}_{I}\left(f^{\prime}\right)\right] \mathbb{E}\left[\tilde{n}_{J}(\nu) \tilde{n}_{J}^{*}\left(\nu^{\prime}\right)\right] \\
& \cdot \tilde{Q}(\nu) \delta(\nu-f) \tilde{Q}^{*}\left(\nu^{\prime}\right) \delta\left(\nu^{\prime}-f^{\prime}\right) d \nu^{\prime} d \nu d f^{\prime} d f \\
= & \int \mathbb{E}\left[\tilde{n}_{I}^{*}(f) \tilde{n}_{I}\left(f^{\prime}\right)\right] \mathbb{E}\left[\tilde{n}_{J}(f) \tilde{n}_{J}^{*}\left(f^{\prime}\right)\right] \tilde{Q}(f) \tilde{Q}^{*}\left(f^{\prime}\right) d f^{\prime} d f .
\end{aligned}
$$

Now, we use that different frequencies in the noise are not correlated to each other and the definition of the twosided noise power spectral density:

$$
\mathbb{E}\left[\tilde{n}_{I}^{*}(f) \tilde{n}_{I}\left(f^{\prime}\right)\right]=: \frac{1}{2} P_{I}\left(\left|f^{\prime}\right|\right) \delta\left(f^{\prime}-f\right)
$$

If we would carelessly plug in this identity, we would get a multiplication of two delta distributions, which is not definable. But we cannot take the expectation of the noise squared over an infinite time integral anyway. So, the delta distribution is actually a $\delta_{T_{I}}$. This is a smooth function and not a distribution and can therefore be multiplied with another $\delta_{T_{J}}$,

$$
\begin{aligned}
\sigma^{2}= & \frac{1}{4} \int P_{I}\left(\left|f^{\prime}\right|\right) P_{J}\left(\left|f^{\prime}\right|\right) \tilde{Q}(f) \tilde{Q}^{*}\left(f^{\prime}\right) \\
& \cdot \int_{-T_{I} / 2}^{T_{I} / 2} e^{-2 \pi i\left(f^{\prime}-f\right) t} d t \int_{-T_{J} / 2}^{T_{J} / 2} e^{2 \pi i\left(f^{\prime}-f\right) t^{\prime}} d t^{\prime} d f^{\prime} d f \\
= & \frac{1}{4} \int P_{I}\left(\left|f^{\prime}\right|\right) P_{J}\left(\left|f^{\prime}\right|\right) \tilde{Q}(f) \tilde{Q}^{*}\left(f^{\prime}\right) \\
& \cdot \int_{-T_{I} / 2}^{T_{I} / 2} \int_{-T_{J} / 2}^{T_{J} / 2} e^{2 \pi i\left(f^{\prime}-f\right)\left(t^{\prime}-t\right)} d t^{\prime} d t d f^{\prime} d f .
\end{aligned}
$$

To evaluate the time integrals, we have to split the integration domain into three regions as depicted in Fig. 19, since we need an integration region that is symmetric around $t^{\prime}-t=0$, where we can use Eq. (C7). The rest can be evaluated separately.

Let $T_{I}<T_{J}, \Delta T=T_{J}-T_{I}$, and $\Delta f=f^{\prime}-f$; then, the time integrals read

$$
\begin{aligned}
\delta_{T_{I}} \delta_{T_{J}} & =\int_{-T_{I} / 2}^{T_{I} / 2} \int_{-T_{J} / 2}^{-T_{I} / 2} e^{-2 \pi i \Delta f\left(t^{\prime}-t\right)} d t^{\prime} \\
& +\int_{-T_{I} / 2}^{T_{I} / 2} e^{-2 \pi i \Delta f\left(t^{\prime}-t\right)} d t^{\prime}+\int_{T_{I} / 2}^{T_{J} / 2} e^{-2 \pi i \Delta f\left(t^{\prime}-t\right)} d t^{\prime} d t
\end{aligned}
$$

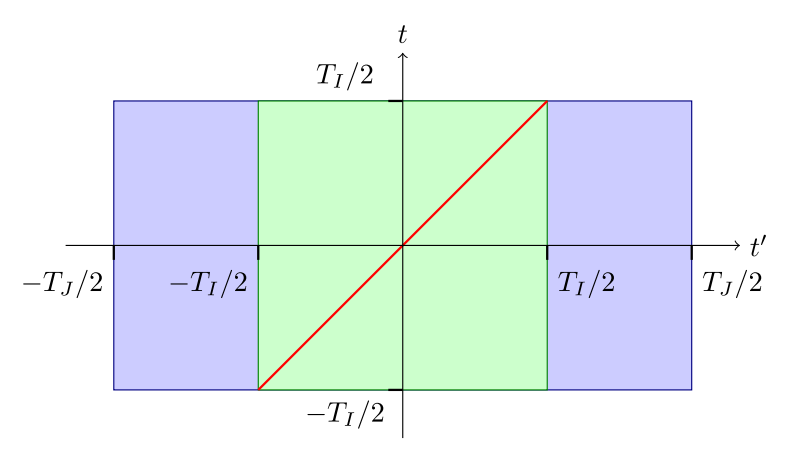

FIG. 19. The green region is symmetric around $t^{\prime}-t=0$ (red line). The blue rectangle marks the entire integration region. 
We substitute $\eta=-t^{\prime}$ in the first integral over $t^{\prime}$, to bring it into the same form as the third one,

$$
\begin{aligned}
\delta_{T_{I}} \delta_{T_{J}} & =\int_{-T_{I} / 2}^{T_{I} / 2}-\int_{T_{J} / 2}^{T_{I} / 2} e^{2 \pi i \Delta f(\eta+t)} d \eta+\int_{T_{I} / 2}^{T_{J} / 2} e^{-2 \pi i \Delta f\left(t^{\prime}-t\right)} d t^{\prime} d t+\int_{-T_{I} / 2}^{T_{I} / 2} \int_{-T_{I} / 2}^{T_{I} / 2} e^{-2 \pi i \Delta f\left(t^{\prime}-t\right)} d t^{\prime} d t \\
& =\int_{-T_{I} / 2}^{T_{I} / 2} e^{2 \pi i \Delta f t} \int_{T_{I} / 2}^{T_{J} / 2} e^{2 \pi i \Delta f t^{\prime}}+e^{-2 \pi i \Delta f t^{\prime}} d t^{\prime} d t+\delta_{T_{I}}^{2}(\Delta f) \\
& =\int_{-T_{I} / 2}^{T_{I} / 2} e^{2 \pi i \Delta f t} d t \int_{T_{I} / 2}^{T_{J} / 2} 2 \cos \left(2 \pi \Delta f t^{\prime}\right) d t^{\prime}+\delta_{T_{I}}^{2}(\Delta f) \\
& =\delta_{T_{I}}(\Delta f)\left(\frac{\sin \left(\pi \Delta f T_{J}\right)-\sin \left(\pi \Delta f T_{I}\right)}{\pi \Delta f}+\delta_{T_{I}}(\Delta f)\right) \\
& \approx \delta\left(f^{\prime}-f\right)\left(\frac{\sin \left(\pi \Delta f T_{I}\right)+\pi \Delta f \Delta T+\mathcal{O}\left((\pi \Delta f \Delta T)^{2}\right)-\sin \left(\pi \Delta f T_{I}\right)}{\pi \Delta f}+\delta_{T_{I}}(\Delta f)\right),
\end{aligned}
$$

where we assumed that $\Delta T \ll T_{I}$.

This approximated distribution acts on functions as

$$
\begin{aligned}
\int g\left(f^{\prime}\right) \delta_{T_{I}} \delta_{T_{J}}(\Delta f) d f^{\prime} & \approx \int g\left(f^{\prime}\right)\left(\Delta T+\mathcal{O}\left(f^{\prime}-f\right)+\delta_{T_{I}}\left(f^{\prime}-f\right)\right) \delta\left(f^{\prime}-f\right) d f \\
& =g(f)\left(T_{I}+\Delta T\right), \quad \forall g \in C^{\infty}(\mathbb{C}) .
\end{aligned}
$$

Inserting this into the variance and integrating over $f^{\prime}$, we get

$$
\sigma^{2}=\frac{1}{4} \int P_{I}\left(\left|f^{\prime}\right|\right) P_{J}\left(\left|f^{\prime}\right|\right) \tilde{Q}(f) \tilde{Q}^{*}\left(f^{\prime}\right) \delta_{T_{I}} \delta_{T_{J}}\left(f^{\prime}-f\right) d f^{\prime} d f=\frac{T_{I}+\Delta T}{4} \int P_{I}(|f|) P_{J}(|f|)|\tilde{Q}(f)|^{2} d f
$$

Using matched filtering with the scalar product, $(\tilde{A}, \tilde{B}):=\int \tilde{A}^{*}(f) \tilde{B}(f) P_{I}(|f|) P_{J}(|f|) d f$, leads us to a filter function:

$$
\tilde{Q}(f)=\frac{\tilde{h}_{I}^{*}(f) \tilde{h}_{J}(f) e^{2 \pi i f \frac{\hat{\Omega} \cdot \Delta \vec{I}_{I J}}{c}}}{P_{I}(|f|) P_{J}(|f|)} .
$$

We can write the signal and noise in terms of the filter function and arrive at the signal-to-noise ratio:

$$
\mathrm{SNR}=\frac{\mu}{\sigma}=\frac{(\tilde{Q}, \tilde{Q})}{\sqrt{\frac{T_{I}+\Delta T}{4}(\tilde{Q}, \tilde{Q})}}=2 \sqrt{\frac{1}{T_{I}+\Delta T} \int \frac{\left|\tilde{h}_{I}(f) \tilde{h}_{J}(f)\right|^{2}}{P_{I}(|f|) P_{J}(|f|)} d f}
$$

We now model the merger as a periodic source, which stops radiating at the end of the merging event at its time coordinate $t_{0}$. Under the assumption that the detectors are far away from the source, we can model the incoming wave as a plane wave with amplitude $h_{0}$ and frequency $f_{0}$, traveling in direction $\hat{\Omega}$ :

$$
h(t)=h_{0} e^{2 \pi i f_{0}\left(t-\frac{\hat{\Omega} \cdot \vec{x}}{c}\right)} \theta\left(t_{0}-\frac{\hat{\Omega} \cdot \vec{x}}{c}-t\right) .
$$

The detector $I$ will measure the signal over a time period $T_{I}$, and the Fourier transform of the measured signal is therefore

$$
\tilde{h}_{I}(f)=\int_{-T_{I} / 2}^{T_{I} / 2} h_{0} e^{2 \pi i f_{0}\left(t-\frac{\hat{\hat{\Omega}} \cdot \vec{x}}{c}\right)} e^{-2 \pi i f t} d t=h_{0} e^{-2 \pi i f_{0} \frac{\hat{\mathrm{s} \cdot \vec{x}}}{c}} \int_{-T_{I} / 2}^{T_{I} / 2} e^{-2 \pi i\left(f-f_{0}\right) t} d t .
$$


Again, we cannot approximate this with a delta distribution; if we did, we would get a $\delta^{4}$ for the $\left|\tilde{h}_{I} \tilde{h}_{J}\right|^{2}$ term,

$$
\left|\tilde{h}_{I}(f) \tilde{h}_{J}(f)\right|^{2}=h_{0}^{4} \int_{-T_{I} / 2}^{T_{I} / 2} \int_{-T_{I} / 2}^{T_{I} / 2} \int_{-T_{J} / 2}^{T_{J} / 2} \int_{-T_{J} / 2}^{T_{J} / 2} e^{-2 \pi i\left(f-f_{0}\right)\left(t^{\prime}-t+\tau^{\prime}-\tau\right)} d \tau^{\prime} d \tau d t^{\prime} d t
$$

We do the same splitting of the $T_{J}$ interval as above, under the assumption $T_{I}<T_{J}$ and using the shorthand $\rho=t^{\prime}-t+\tau^{\prime}-\tau$ :

$$
\begin{aligned}
\left|\tilde{h}_{I} \tilde{h}_{J}\right|^{2} \propto \delta_{T_{I}}^{2} \delta_{T_{J}}^{2}= & \int_{-T_{I} / 2}^{T_{I} / 2} \int_{-T_{J} / 2}^{T_{J} / 2} e^{-2 \pi i\left(f^{\prime}-f\right)\left(t^{\prime}-t+\tau^{\prime}-\tau\right)} d \tau^{\prime} d \tau d t^{\prime} d t \\
= & \int_{-T_{I} / 2}^{T_{I} / 2} \int_{-T_{J} / 2}^{-T_{I} / 2} e^{-2 \pi i \Delta f \rho} d \tau^{\prime} d \tau+\int_{-T_{I} / 2}^{T_{I} / 2} e^{-2 \pi i \Delta f \rho} d \tau^{\prime} d \tau+\int_{T_{I} / 2}^{T_{J} / 2} e^{-2 \pi i \Delta f \rho} d \tau^{\prime} d \tau d t^{\prime} d t \\
= & \int_{-T_{I} / 2}^{T_{I} / 2} e^{-2 \pi i \Delta f\left(t^{\prime}-t\right)} d t^{\prime} d t \int_{T_{I} / 2}^{T_{J} / 2} e^{2 \pi i \Delta f\left(\tau^{\prime}-\tau\right)}+e^{-2 \pi i \Delta f\left(\tau^{\prime}-\tau\right)} d \tau^{\prime} d \tau+\int_{-T_{I} / 2}^{T_{I} / 2} e^{-2 \pi i \Delta f \rho} d \tau^{\prime} d \tau d t^{\prime} d t \\
= & \delta_{T_{I}}^{2}(\Delta f) \int_{T_{I} / 2}^{T_{J} / 2} 2 \cos \left(2 \pi \Delta f\left(\tau^{\prime}-\tau\right)\right) d \tau^{\prime} d \tau+\delta_{T_{I}}^{4}(\Delta f) \\
= & \delta_{T_{I}}^{2}(\Delta f)\left(\frac{1}{\pi \Delta f} \int_{T_{I} / 2}^{T_{J} / 2} \sin \left(\pi \Delta f\left(T_{J}-2 \tau\right)\right)-\sin \left(\pi \Delta f\left(T_{I}-2 \tau\right)\right) d \tau+\delta_{T_{I}}^{2}(\Delta f)\right) \\
= & \delta_{T_{I}}^{2}(\Delta f)\left(-\frac{1}{2(\pi \Delta f)^{2}}\left\{\cos \left(\pi \Delta f\left(T_{J}-T_{J}\right)\right)-\cos \left(\pi \Delta f\left(T_{J}-T_{I}\right)\right)\right.\right. \\
& \left.\left.-\cos \left(\pi \Delta f\left(T_{I}-T_{J}\right)\right)+\cos \left(\pi \Delta f\left(T_{I}-T_{I}\right)\right)\right\}+\delta_{T_{I}}^{2}(\Delta f)\right) \\
= & \delta_{T_{I}}^{2}(\Delta f)\left(\frac{1}{(\pi \Delta f)^{2}}\{\cos (\pi \Delta f \Delta T)-1\}+\delta_{T_{I}}^{2}(\Delta f)\right) \\
= & \delta\left(f^{\prime}-f\right) \delta_{T_{I}}(\Delta f)\left(\frac{1}{(\pi \Delta f)^{2}}\left\{1-\frac{1}{2}(\pi \Delta f \Delta T)^{2}+\mathcal{O}\left(\Delta f^{4}\right)-1\right\}+\delta_{T_{I}}^{2}(\Delta f)\right) .
\end{aligned}
$$

The action on a function $g \in C^{\infty}(\mathbb{C})$ is

$$
\begin{aligned}
\int & g\left(f^{\prime}\right) \delta_{T_{I}}^{2} \delta_{T_{J}}^{2}\left(f^{\prime}-f\right) d f^{\prime} \\
= & \int g\left(f^{\prime}\right) \delta_{T_{I}}(\Delta f)\left(\frac{\Delta T^{2}}{2}+\mathcal{O}\left(\Delta f^{2}\right)+\delta_{T_{I}}(\Delta f)^{2}\right) \\
& \times \delta\left(f^{\prime}-f\right) d f^{\prime} \\
= & g(f) \delta_{T_{I}}(0)\left(\frac{\Delta T^{2}}{2}+\delta_{T_{I}}(0)^{2}\right) \\
= & g(f) T_{I}\left(\frac{\Delta T^{2}}{2}+T_{I}^{2}\right) .
\end{aligned}
$$

When we plug this into the SNR, we get

$$
\begin{aligned}
\mathrm{SNR} & =2 \sqrt{\frac{T_{I}}{T_{I}+\Delta T}\left(\frac{\Delta T^{2}}{2}+T_{I}^{2}\right) \frac{h_{0}^{4}}{P_{I}\left(\left|f_{0}\right|\right) P_{J}\left(\left|f_{0}\right|\right)}} \\
& \approx 2\left(T_{I}+\frac{\hat{\Omega} \cdot \Delta \vec{x}_{I J}}{2 c}\right) \frac{h_{0}^{2}}{\sqrt{P_{I}\left(f_{0}\right) P_{J}\left(f_{0}\right)}},
\end{aligned}
$$

where we used the identification of the integration time with the direction of the source in Eq. (C1).
The minimal amplitude is then given by

$$
\begin{aligned}
h_{\min } & =\sqrt{32}\left(\frac{\left(T_{I}+\Delta T\right) P_{I}(f) P_{J}(f)}{T_{I}\left(\frac{\Delta T^{2}}{2}+T_{I}^{2}\right)}\right)^{1 / 4} \\
& \approx \sqrt{32}\left(\frac{1}{\sqrt{T_{I}}}+\frac{\hat{\Omega} \cdot \Delta \vec{x}_{I J}}{4 c{\sqrt{T_{I}}}^{3}}\right) \sqrt[4]{P_{I}(f) P_{J}(f)} .
\end{aligned}
$$

Including polarizations, we have a gravitational wave $h_{i j}(t)$, which induces the signal $h_{I}(t)$ in detector $I$,

$$
h_{i j}(t)=\sum_{A} h_{A} e^{2 \pi i f_{0}\left(t-\frac{\hat{\Omega} \vec{x}}{c}\right)+\varphi_{A}} \theta\left(t_{0}-\frac{\hat{\Omega} \cdot \vec{x}}{c}-t\right) e_{i j}^{A}
$$

$$
h_{I}(t)=\sum_{A} h_{A} F_{I}^{A}(\hat{\Omega}) e^{2 \pi i f_{0}\left(t-\frac{\hat{\mathbf{S}} \cdot \vec{x}_{I}}{c}\right)+\varphi_{A}} \theta\left(t_{0}-\frac{\hat{\Omega} \cdot \vec{x}_{I}}{c}-t\right),
$$

where $h_{A}$ is the amplitude of the wave in polarization $A$ and $\varphi_{A}$ accounts for the fact that the polarizations could be phase shifted. 
For the absolute value squared of the cross-correlated signals of two detectors, we get

$$
\begin{aligned}
\left|\tilde{h}_{I}(f) \tilde{h}_{J}(f)\right|^{2} & =\left|\tilde{h}_{I}(f)\right|^{2}\left|\tilde{h}_{J}(f)\right|^{2} \\
& =\left|\sum_{A} h_{A} F_{I}^{A}(\hat{\Omega}) e^{-\frac{2 \pi i f_{0}}{c} \hat{\Omega} \cdot \vec{x}_{I}+i \varphi_{A}}\right|^{2}\left|\sum_{A} h_{A} F_{J}^{A}(\hat{\Omega}) e^{-\frac{2 \pi i f_{0}}{c} \hat{\Omega} \cdot \vec{x}_{J}+i \varphi_{A}}\right|^{2} \int_{-T_{I} / 2}^{T_{I} / 2} \int_{-T_{J} / 2}^{T_{J} / 2} e^{-2 \pi i\left(f-f_{0}\right) \rho} d^{4} \rho \\
& =\left|\sum_{A} h_{A} F_{I}^{A}(\hat{\Omega}) e^{i \varphi_{A}}\right|^{2}\left|\sum_{A} h_{A} F_{J}^{A}(\hat{\Omega}) e^{i \varphi_{A}}\right|^{2} \delta_{T_{I}}\left(f-f_{0}\right)\left(\frac{\Delta T^{2}}{2}+\delta_{T_{I}}\left(f-f_{0}\right)^{2}\right) .
\end{aligned}
$$

We make the assumption that the gravitational wave has only one of the polarizations $h=\sum_{A^{\prime}} h_{A^{\prime}} \delta_{A^{\prime} A}$, to get the signal-tonoise ratio for that polarization,

$$
\begin{aligned}
\mathrm{SNR}_{A} & :=\left.\frac{\mu}{\sigma}\right|_{h=h_{A}} \\
& =2 \sqrt{\frac{T_{I}}{T_{I}+\Delta T}\left(\frac{\Delta T^{2}}{2}+T_{I}^{2}\right) \frac{\left(\left|h_{A}\right|^{2} F_{I}^{A}(\hat{\Omega}) F_{J}^{A}(\hat{\Omega})\right)^{2}}{P_{I}\left(\left|f_{0}\right|\right) P_{J}\left(\left|f_{0}\right|\right)}} \\
& \approx 2\left(T_{I}+\frac{\hat{\Omega} \cdot \Delta \vec{x}_{I J}}{2 c}\right) \frac{\left|h_{A}\right|^{2} F_{I}^{A}(\hat{\Omega}) F_{J}^{A}(\hat{\Omega})}{\sqrt{P_{I}\left(f_{0}\right) P_{J}\left(f_{0}\right)}}
\end{aligned}
$$

which we get by replacing $h_{0}^{4} \mapsto\left(\left|h_{A}\right|^{2} F_{I}^{A}(\hat{\Omega}) F_{J}^{A}(\hat{\Omega})\right)^{2}$ in Eq. (C26).

For multiple detectors, we use the maximum likelihood method and calculate the Fisher matrix. The likelihood function is given by

$$
L\left(\mu_{I J}, \vec{\theta}\right)=e^{-\sum_{(I, J)} \frac{\left(Y_{I J}-\mu_{I J}\right)^{2}}{2 \sigma_{I J}^{2}}},
$$

where $\mu_{I J}=\mathbb{E}\left[Y_{I J}\right]$ is the ensemble average of the correlated signals of the detectors $I$ and $J$. Its variance $\sigma_{I J}^{2}=\mathbb{V}\left[Y_{I J}\right]$ is given by Eq. (C18) without the filtering. Multiplying the SNR in Eq. (C26) with the noise, we get

$$
\mu_{I J}=\sqrt{T_{I}\left(\frac{\Delta T^{2}}{2}+T_{I}^{2}\right)}\left|\tilde{h}_{I} \tilde{h}_{J}\right|
$$

The matrix element $F_{A A^{\prime}}$ of the Fisher matrix is then given by

$$
\begin{aligned}
& F_{A A^{\prime}}=\mathbb{E}\left[\left(\partial_{\left|h_{A}\right|^{2}} \ln L\right)\left(\partial_{\left|h_{A^{\prime}}\right|^{2}} \ln L\right)\right] \\
& =\mathbb{E}\left[\left(\sum_{(I, J)} \frac{1}{\sigma_{I J}^{2}}\left(Y_{I J}-\mu_{I J}\right) \sqrt{T_{I}\left(\frac{\Delta T^{2}}{2}+T_{I}^{2}\right)}\right)^{2}\left(\partial_{\left|h_{A}\right|}\left|\tilde{h}_{I} \tilde{h}_{J}\right|\right)\left(\partial_{\left|h_{A^{\prime}}\right|}\left|\tilde{h}_{I} \tilde{h}_{J}\right|\right)\right]
\end{aligned}
$$

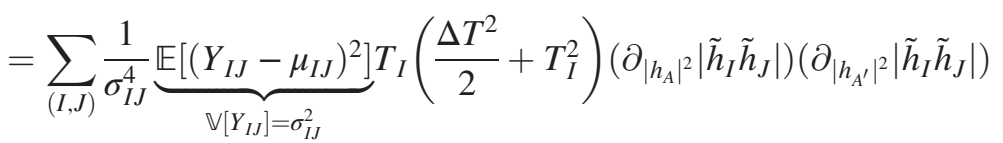

$$
\begin{aligned}
& +\sum_{(I, J) \neq\left(I^{\prime}, J^{\prime}\right)} \frac{1}{\sigma_{I J}^{2} \sigma_{I^{\prime} J^{\prime}}^{2}} \underbrace{\mathbb{E}\left[\left(Y_{I J}-\mu_{I J}\right)\left(Y_{I^{\prime} J^{\prime}}-\mu_{I^{\prime} J^{\prime}}\right)\right]}_{\operatorname{Cov}\left(Y_{I J}, Y_{I^{\prime} J^{\prime}}\right)=0} T_{I}\left(\frac{\Delta T^{2}}{2}+T_{I}^{2}\right)\left(\partial_{\left|h_{A}\right|^{2}}\left|\tilde{h}_{I} \tilde{h}_{J}\right|\right)\left(\partial_{\left|h_{A^{\prime}}\right|^{2}}\left|\tilde{h}_{I^{\prime}} \tilde{h}_{J^{\prime}}\right|\right) \\
& =\sum_{(I, J)} \frac{4 T_{I}}{\left(T_{I}+\Delta T\right) P_{I} P_{J}}\left(\frac{\Delta T^{2}}{2}+T_{I}^{2}\right)\left(\partial_{\left|h_{A}\right|^{2}}\left|\tilde{h}_{I} \tilde{h}_{J}\right|\right)\left(\partial_{\left|h_{A^{\prime}}\right|}\left|\tilde{h}_{I} \tilde{h}_{J}\right|\right) \text {. }
\end{aligned}
$$

The SNR squared of a specific polarization $A$ is defined by dividing the square of the quantity we are looking for $\left|h_{A}\right|^{2}$ by its variance $\sigma_{A}$, under the condition that the incoming wave has only that polarization,

$$
\operatorname{SNR}_{A}^{2}:=\left.\frac{\left(\left|h_{A}\right|^{2}\right)^{2}}{\sigma_{A}^{2}}\right|_{h=h_{A}}=\left.\frac{\left(\left|h_{A}\right|^{2}\right)^{2}}{\left(F^{-1}\right)_{A A}}\right|_{h=h_{A}}=\left.\frac{\left(\left|h_{A}\right|^{2}\right)^{2} \operatorname{det} \mathbf{F}}{\mathcal{F}_{A}}\right|_{h=h_{A}} .
$$




\section{APPENDIX D: FISHER MATRIX ENTRIES}

As can be seen in Appendix C, the Fisher matrix can be written as a sum of Fisher matrices of single detector pairs, which consist of a prefactor and two derivative terms for the row and column of the entry. If $\theta_{i, j}$ are polarizations, we have

$$
F_{i j} \propto\left(\partial_{\theta_{i}}\left|\tilde{h}_{I} \tilde{h}_{J}\right|\right)\left(\partial_{\theta_{j}}\left|\tilde{h}_{I} \tilde{h}_{J}\right|\right)
$$

where $\vec{\theta}=(\theta, \phi,+, \times, x, y, b, l)$ are the parameters we are looking for.
Here, we calculate those derivative terms. We start by writing out the absolute value squared of the correlation signal,

$$
\left|\tilde{h}_{I} \tilde{h}_{J}\right|^{2}=\left|\sum_{A} h_{A} F_{I}^{A} \sum_{A^{\prime}} h_{A^{\prime}} F_{J}^{A^{\prime}}\right|^{2}
$$

where the phase $\varphi_{A}$ of the polarization $A$ is integrated in the complex valued amplitude $h_{A} \in \mathbb{C}$.

We split the multiplied signals up into sums over terms in which the polarizations coincide and in which they are different:

$$
\begin{aligned}
\left|\tilde{h}_{I} \tilde{h}_{J}\right|^{2}= & \left|\sum_{A} h_{A}^{2} F_{I}^{A} F_{J}^{A}+\sum_{A \neq A^{\prime}} h_{A} h_{A^{\prime}} F_{I}^{A} F_{J}^{A^{\prime}}\right|^{2} \\
= & \left(\sum_{A} h_{A}^{2} F_{I}^{A} F_{J}^{A}\right)\left(\sum_{B} h_{B}^{2} F_{I}^{B} F_{J}^{B}\right)^{*}+\left(\sum_{A} h_{A}^{2} F_{I}^{A} F_{J}^{A}\right)\left(\sum_{B \neq B^{\prime}} h_{B} h_{B^{\prime}} F_{I}^{B} F_{J}^{B^{\prime}}\right)^{*} \\
& +\left(\sum_{A \neq A^{\prime}} h_{A} h_{A^{\prime}} F_{I}^{A} F_{J}^{A^{\prime}}\right)\left(\sum_{B} h_{B}^{2} F_{I}^{B} F_{J}^{B}\right)^{*}+\left(\sum_{A \neq A^{\prime}} h_{A} h_{A^{\prime}} F_{I}^{A} F_{J}^{A^{\prime}}\right)\left(\sum_{B \neq B^{\prime}} h_{B} h_{B^{\prime}} F_{I}^{B} F_{J}^{B^{\prime}}\right)^{*} \\
= & \sum_{A}\left(\left|h_{A}\right|^{2} F_{I}^{A} F_{J}^{A}\right)^{2}+\sum_{A \neq B}\left(h_{A} h_{B}^{*}\right)^{2} F_{I}^{A} F_{J}^{A} F_{I}^{B} F_{J}^{B} \\
& +\sum_{A} h_{A}\left|h_{A}\right|^{2} F_{I}^{A} F_{J}^{A} \sum_{B \neq A} h_{B}^{*}\left(F_{I}^{A} F_{J}^{B}+F_{I}^{B} F_{J}^{A}\right)+\sum_{A \neq B \neq B^{\prime}} h_{A}^{2} F_{I}^{A} F_{J}^{A} h_{B}^{*} h_{B^{\prime}}^{*} F_{I}^{B} F_{J}^{B^{\prime}} \\
& +\sum_{B} h_{B}^{*}\left|h_{B}\right|^{2} F_{I}^{B} F_{J}^{B} \sum_{A \neq B} h_{A}\left(F_{I}^{B} F_{J}^{A}+F_{I}^{A} F_{J}^{B}\right)+\sum_{A \neq A^{\prime} \neq B} h_{A} h_{A^{\prime}} F_{I}^{A} F_{J}^{A^{\prime}}\left(h_{B}^{*}\right)^{2} F_{I}^{B} F_{J}^{B} \\
& +\sum_{A \neq A^{\prime}}\left|h_{A}\right|^{2}\left|h_{A^{\prime}}\right|^{2}\left[\left(F_{I}^{A} F_{J}^{A^{\prime}}\right)^{2}+F_{I}^{A} F_{J}^{A^{\prime}} F_{I}^{A^{\prime}} F_{J}^{A}\right] \\
& +\sum_{A}\left|h_{A}\right|^{2} \sum_{B \neq B^{\prime}} h_{B} h_{B^{\prime}}^{*}\left[\left(F_{I}^{A}\right)^{2} F_{J}^{B} F_{J}^{B^{\prime}}+F_{I}^{A} F_{J}^{A}\left(F_{I}^{B} F_{J}^{B^{\prime}}+F_{I}^{B^{\prime}} F_{J}^{B}\right)+\left(F_{J}^{A}\right)^{2} F_{I}^{B} F_{I}^{B^{\prime}}\right] \\
& +\sum_{A \neq A^{\prime} \neq B \neq B^{\prime}} h_{A} h_{A^{\prime}} h_{B}^{*} h_{B^{\prime}}^{*} F_{I}^{A} F_{J}^{A^{\prime}} F_{I}^{B} F_{J}^{B^{\prime}} .
\end{aligned}
$$

When we take the derivative after $\left|h_{A}\right|^{2}$, all sums that do not contain such a term vanish:

$$
\begin{aligned}
\partial_{\left.|h A|\right|^{\mid}}\left|\tilde{h}_{I} \tilde{h}_{J}\right|= & \frac{1}{2 \sqrt{\left|\tilde{h}_{I} \tilde{h}_{J}\right|}}\left\{2\left|h_{A}\right|^{2}\left(F_{I}^{A} F_{J}^{A}\right)^{2}+h_{A} F_{I}^{A} F_{J}^{A} \sum_{B \neq A} h_{B}^{*}\left(F_{I}^{A} F_{J}^{B}+F_{I}^{B} F_{J}^{A}\right)\right. \\
& +h_{A}^{*} F_{I}^{A} F_{J}^{A} \sum_{B \neq A} h_{B}\left(F_{I}^{A} F_{J}^{B}+F_{I}^{B} F_{J}^{A}\right)+\sum_{A^{\prime} \neq A}\left|h_{A^{\prime}}\right|^{2}\left[\left(F_{I}^{A} F_{J}^{A^{\prime}}\right)^{2}+2 F_{I}^{A} F_{J}^{A^{\prime}} F_{I}^{A^{\prime}} F_{J}^{A}+\left(F_{I}^{A^{\prime}} F_{J}^{A}\right)^{2}\right] \\
& +\sum_{\substack{B \neq B^{\prime} \\
B, B^{\prime} \neq A}} h_{B} h_{B^{\prime}}^{*}\left[\left(F_{I}^{A}\right)^{2} F_{J}^{B} F_{J}^{B^{\prime}}+F_{I}^{A} F_{J}^{A}\left(F_{I}^{B} F_{J}^{B^{\prime}}+F_{I}^{B^{\prime}} F_{J}^{B}\right)+\left(F_{J}^{A}\right)^{2} F_{I}^{B} F_{I}^{\left.\left.B^{\prime}\right]\right\}} .\right.
\end{aligned}
$$

We add the condition that we have an incoming wave with polarization $A_{0}$, and therefore all terms proportional to two different polarizations are zero: 


$$
\begin{aligned}
\left.\partial_{\left|h_{A}\right|}\left|\tilde{h}_{I} \tilde{h}_{J}\right|\right|_{h=h_{A_{0}}} & =\frac{1}{2 \sqrt{\left(\left|h_{A_{0}}\right|^{2} F_{I}^{A_{0}} F_{J}^{A_{0}}\right)^{2}+0}}\left\{2 \delta_{A A_{0}}\left|h_{A}\right|^{2}\left(F_{I}^{A} F_{J}^{A}\right)^{2}+\left(1-\delta_{A A_{0}}\right)\left|h_{A_{0}}\right|^{2}\left[\left(F_{I}^{A} F_{J}^{A_{0}}\right)^{2}+2 F_{I}^{A} F_{J}^{A_{0}} F_{I}^{A_{0}} F_{J}^{A}+\left(F_{I}^{A_{0}} F_{J}^{A}\right)^{2}\right]\right\} \\
& =F_{I}^{A} F_{J}^{A}+\left(1-\delta_{A A_{0}}\right) \frac{1}{2}\left[\frac{\left(F_{I}^{A}\right)^{2} F_{J}^{A_{0}}}{F_{I}^{A_{0}}}+\frac{\left(F_{J}^{A}\right)^{2} F_{I}^{A_{0}}}{F_{J}^{A_{0}}}\right]
\end{aligned}
$$

If we calculate a matrix element in the $\theta$ or $\phi$ row or column, we cannot pull the term $\sqrt{T_{I}\left(\frac{\Delta T^{2}}{2}+T_{I}^{2}\right)}$ out in front, so the general Fisher matrix element looks like

$$
F_{i j}=\mathbb{E}\left[\left(\partial_{\theta_{i}} \ln L\right)\left(\partial_{\theta_{j}} \ln L\right)\right]=\sum_{(I, J)} \frac{1}{\sigma_{I J}^{2}}\left(\partial_{\theta_{i}} \sqrt{T_{I}\left(\frac{\Delta T^{2}}{2}+T_{I}^{2}\right)}\left|\tilde{h}_{I} \tilde{h}_{J}\right|\right) \cdot\left(\partial_{\theta_{j}} \sqrt{T_{I}\left(\frac{\Delta T^{2}}{2}+T_{I}^{2}\right)}\left|\tilde{h}_{I} \tilde{h}_{J}\right|\right) .
$$

The variance of the true signal $Y_{I J}$ is dependent on the true time difference, and we can treat it as a parameter when we take the derivative after the estimated $\theta$ value,

$$
\mathbb{V}\left[Y_{I J}\right]=\sigma_{I J}^{2}=\frac{T_{I}+\Delta T}{4} P_{I} P_{J}, \quad \Delta T=\frac{\hat{\omega} \cdot \Delta \vec{x}_{I J}}{c},
$$

where $\hat{\omega}$ is the true direction of the source.

The derivative term for the angle $\theta$ for a wave with polarization $A_{0}$ is given by

$\left.\partial_{\theta} \sqrt{T_{I}\left(\frac{\Delta T^{2}}{2}+T_{I}^{2}\right)}\left|\tilde{h}_{I} \tilde{h}_{J}\right|\right|_{h=h_{A_{0}}}=\frac{T_{I} \Delta T}{2 \sqrt{T_{I}\left(\frac{\Delta T^{2}}{2}+T_{I}^{2}\right)}} \frac{\hat{\Omega}_{, \theta} \cdot \Delta \vec{x}_{I J}}{c}\left|h_{A_{0}}\right|^{2} F_{I}^{A_{0}} F_{J}^{A_{0}}+\sqrt{T_{I}\left(\frac{\Delta T^{2}}{2}+T_{I}^{2}\right)}\left|h_{A_{0}}\right|^{2}\left(F_{I, \theta}^{A_{0}} F_{J}^{A_{0}}+F_{I}^{A_{0}} F_{J, \theta}^{A_{0}}\right)$.

[1] B. P. Abbott et al., Observation of Gravitational Waves from a Binary Black Hole Merger, Phys. Rev. Lett. 116, 061102 (2016).

[2] B. P. Abbott et al., GW151226: Observation of Gravitational Waves from a 22-Solar-Mass Binary Black Hole Coalescence, Phys. Rev. Lett. 116, 241103 (2016).

[3] B. P. Abbott et al., GW170104: Observation of a 50-SolarMass Binary Black Hole Coalescence at Redshift 0.2, Phys. Rev. Lett. 118, 221101 (2017).

[4] B. P. Abbott et al., GW170608: Observation of a 19 solarmass binary black hole coalescence, Astrophys. J. Lett. 851, L35 (2017).

[5] B. P. Abbott et al., GW170814: A Three-Detector Observation of Gravitational Waves from a Binary Black Hole Coalescence, Phys. Rev. Lett. 119, 141101 (2017).

[6] The current state of the detections by the LIGO/VIRGO Collaboration can be found on https://www.ligo.caltech .edu/page/detection-companion-papers.

[7] B. P. Abbott et al., GW170817: Observation of Gravitational Waves from a Binary Neutron Star Inspiral, Phys. Rev. Lett. 119, 161101 (2017).

[8] B. P. Abbott et al., Multi-messenger observations of a binary neutron star merger, Astrophys. J. Lett. 848, 2 (2017).
[9] C. Everitt et al., Gravity Probe B data analysis status and potential for improved accuracy of scientific results, Classical Quantum Gravity 25, 114002 (2008).

[10] P. Touboul et al., The MICROSCOPE mission: First Results of a Space Test of the Equivalence Principle, Phys. Rev. Lett. 119, 231101 (2017).

[11] C. M. Will, The confrontation between general relativity and experiment, Living Rev. Relativity 17, 4 (2014).

[12] KAGRA Collaboration, Construction of KAGRA: An underground gravitational wave observatory, Prog. Theor. Exp. Phys. 2018, 013F01 (2018).

[13] IndIGO Consortium, LIGO-India, Proposal for an interferometric gravitational-wave observatory, 2011, https:// dcc.ligo.org/public/0075/M1100296/002/LIGO-India_lwv2.pdf.

[14] ET Science Team, Einstein gravitational wave Telescope conceptual design study, ET-0106C-10, Issue 4 (2011), https://tds.virgo-gw.eu/?call_file=ET-0106C-10.pdf.

[15] M. Armano et al., Beyond the Required LISA Free-Fall Performance: New LISA Pathfinder Results down to $20 \mu \mathrm{Hz}$, Phys. Rev. Lett. 120, 061101 (2018).

[16] LISA Consortium, LISA: A proposal in response to the ESA call for L3 mission concepts, arXiv:1702.00786. 
[17] S. Kawamura et al., The Japanese space gravitational wave antenna-DECIGO, Classical Quantum Gravity 23, S125 (2006).

[18] K. Yagi and N. Seto, Detector configuration of DECIGO/ BBO and identification of cosmological neutronstar binaries, Phys. Rev. D 83, 044011 (2011).

[19] S. Isoyama, H. Nakano, and T. Nakamura, Multiband gravitational-wave astronomy: Observing binary inspirals with a decihertz detector, B-DECIGO, arXiv:1802.06977v1.

[20] T. Nakamura et al., Pre-DECIGO can get the smoking gun to decide the astrophysical or cosmological origin of GW150914-like binary black holes, Prog. Theor. Exp. Phys. 2016, 093E01 (2016).

[21] A. Nishizawa, A. Taruya, K. Hayama, S. Kawamura, and S. Masa-aki, Probing nontensorial polarizations of stochastic GWB with ground-based laser interferometers, Phys. Rev. D 79, 082002 (2009).
[22] A. Nishizawa, A. Taruya, and S. Kawamura, Cosmological test of gravity with polarizations of stochastic GW around 0.1-1 Hz, Phys. Rev. D 81, 104043 (2010).

[23] B. Allen and J. D. Romano, Detecting a stochastic background of gravitational radiation: Signal processing strategies and sensitivities, Phys. Rev. D 59, 102001 (1999).

[24] M. Maggiore, Gravitational Waves, Theory and Experiments (Oxford University, New York, 2008), Vol. 1.

[25] LIGO Scientific Collaboration, Advanced LIGO, Classical Quantum Gravity 32, 074001 (2015).

[26] D. V. Martynov et al., The sensitivity of the advanced LIGO detectors at the beginning of gravitational wave astronomy, Phys. Rev. D 93, 112004 (2016).

[27] K. M. Górski, B. Wandelt, E. Hivon, F. K. Hansen, and A. J. Banday, The HEALPix primer, http://healpix.sourceforge .net/pdf/intro.pdf, 2018. 